Cite this: Nanoscale, 2014, 6, 6301

\title{
Graphene nanoribbon blends with P3HT for organic electronics $\uparrow$
}

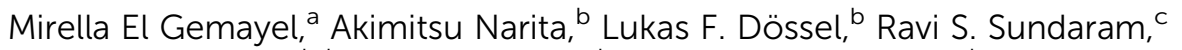

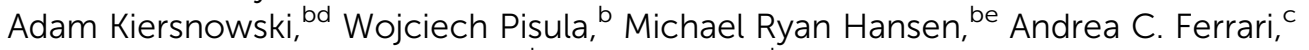 \\ Emanuele Orgiu, ${ }^{a}$ Xinliang Feng, ${ }^{* b}$ Klaus Müllen ${ }^{\star b}$ and Paolo Samorì ${ }^{\star a}$
}

In organic field-effect transistors (OFETs) the electrical characteristics of polymeric semiconducting materials suffer from the presence of structural/morphological defects and grain boundaries as well as amorphous domains within the film, hindering an efficient transport of charges. To improve the percolation of charges we blend a regioregular poly(3-hexylthiophene) (P3HT) with newly designed $N=$ 18 armchair graphene nanoribbons (GNRs). The latter, prepared by a bottom-up solution synthesis, are expected to form solid aggregates which cannot be easily interfaced with metallic electrodes, limiting charge injection at metal-semiconductor interfaces, and are characterized by a finite size, thus by grain boundaries, which negatively affect the charge transport within the film. Both P3HT and GNRs are soluble/dispersible in organic solvents, enabling the use of a single step co-deposition process. The resulting OFETs show a three-fold increase in the charge carrier mobilities in blend films, when compared to pure P3HT devices. This behavior can be ascribed to GNRs, and aggregates thereof, facilitating the transport of the charges within the conduction channel by connecting the domains of the semiconductor film. The electronic characteristics of the devices such as the $I_{\text {on }} / I_{\text {off }}$ ratio are not affected by the addition of GNRs at different loads. Studies of the electrical characteristics under illumination for potential use of our blend films as organic phototransistors (OPTs) reveal a tunable photoresponse. Therefore, our strategy offers a new method towards the enhancement of the performance of OFETs, and holds potential for technological applications in (opto)electronics.

Received 15th January 2014

Accepted 10th February 2014

DOI: $10.1039 / c 4 n r 00256 c$

www.rsc.org/nanoscale

\section{Introduction}

Over the past few years, a great effort has been devoted to the development of new organic semiconducting materials, combining solution processability, stability under ambient conditions, and high charge carrier mobility ${ }^{\mathbf{1 - 1 0}}$ for applications in organic electronics and particularly in organic field-effect transistors (OFETs). OFETs can be fabricated by using either small molecules or polymeric semiconductors as electroactive layers. In the latter case, despite the fact that polydisperse molecules can form extended networks for efficient percolation

${ }^{a}$ Nanochemistry Laboratory, ISIS \& icFRC, Université de Strasbourg \& CNRS, 8 allée Gaspard Monge, 67000 Strasbourg, France.E-mail: samori@unistra.fr

${ }^{b}$ Max-Planck Institute for Polymer Research, Ackermannweg 10, 55124 Mainz, Germany.E-mail: muellen@mpip-mainz.mpg.de; feng@mpip-mainz.mpg.de

${ }^{c}$ Cambridge Graphene Center, Department of Engineering, Cambridge University, $9 \mathrm{JJ}$ Thomson Avenue, Cambridge CB3 OFA, UK

${ }^{d}$ Polymer Engineering and Technology Division Wroclaw University of Technology Wybrzeze Wyspianskiego 27, 50-370 Wroclaw, Poland

${ }^{e}$ Interdisciplinary Nanoscience Center (iNANO) and Department of Chemistry, Aarhus University, Gustav Wieds Vej 14, DK-8000 Aarhus C, Denmark

$\dagger$ Electronic supplementary information (ESI) available. See DOI: 10.1039/c4nr00256c of charges, due to their polycrystalline nature the obtained material is characterized by the presence of structural and morphological defects, grain boundaries and amorphous domains hampering efficient charge transport. ${ }^{\mathbf{1 1}}$ To circumvent this problem, polymeric semiconductors have been blended with either small molecules or other (semi)conducting systems. ${ }^{11-19}$ By blending different components new functions can be conferred to the material and to the device, ${ }^{20}$ benefiting the properties brought about by each component and exceeding its individual performance. Such an effect varies with the degree of phase segregation between the different components in the blend. Amongst polymeric semiconductors, regioregular poly(3hexylthiophene) - P3HT is a prototypical system for solution processed OFETs. ${ }^{21-25}$ The performance of P3HT based OFETs has been improved by co-deposition with graphene, revealing an over 20 times increase in charge carrier mobilities when compared to the pure P3HT. ${ }^{26}$ This enhanced electrical characteristic can be ascribed to the beneficial role of graphene, which builds preferential paths for charge transport, therefore increasing the overall hole mobility, ${ }^{27}$ similar to previous findings in OFETs based on carbon nanotubes with $\mathrm{P} 3 \mathrm{HT}$; $^{\mathbf{2} 29}$ however, this was accompanied by a decrease of the on-off current ratio $\left(I_{\mathrm{on}} / I_{\mathrm{off}}\right)$. 
Due to the fact that graphene has zero band-gap, ${ }^{30}$ with high charge carrier mobilities, ${ }^{31}$ it cannot be used as an electroactive component to be incorporated in the channel of a regular OFET. Significant effort was devoted to opening a band gap in graphene. ${ }^{32}$ One approach was to confine the two dimensional (2d) sheet of graphene creating graphene nanoribbons (GNRs). ${ }^{33}$ Fabrication of GNRs has been predominantly carried out by topdown methods, such as lithographic patterning of graphene sheets, ${ }^{34-36}$ longitudinal unzipping of carbon nanotubes, ${ }^{37-39}$ and chemical extraction from exfoliated graphite. ${ }^{40}$ However, these methods lead to the production of GNRs exhibiting a broad (10-100 nm) width distribution ${ }^{37-39}$ as well as defective edge structures, resulting in GNRs with non-uniform electronic properties, e.g. band gap and ionization potential. In addition, top-down methods for the production of GNRs typically suffer from very low yield, hindering practical applications of the GNRs. ${ }^{37}$ In contrast, a bottom-up synthetic approach based on solution-mediated ${ }^{\mathbf{4 1 - 4 4}}$ and surface-assisted ${ }^{45}$ cyclodehydrogenation $^{\mathbf{4 6 7}}$ of tailor-made polyphenylene precursors has been developed, enabling the fabrication of a variety of uniform and structurally defined GNRs with a lateral width of ca. 1-2 nm, which possess large and defined band gaps (1.1-1.7 eV). ${ }^{42,45}$ By using the solution-mediated method it is possible to produce GNRs in a large scale with a relatively high yield, as well as to render the GNRs dispersible in organic solvents by installing solubilizing groups at the peripheral positions. ${ }^{\mathbf{4 1 , 4 2 , 4 4}}$ It was calculated that the absolute value of the valence band, which is related to the ionization potential, decreases as the GNRs extend laterally. ${ }^{48}$ We have recently reported laterally extended GNRs with a width of 1.54-1.98 nm, but the absolute value of the valence band of these GNRs was estimated to be still higher than that of P3HT, hindering the use of these GNRs for blending with P3HT. ${ }^{42,48}$ Further, the edges of these GNRs contain a cove-type structure, and the fabrication of broad $(\sim 2$ $\mathrm{nm}$ ) and fully armchair edged GNRs has remained a challenge.

In this work we present the solution synthesis of $N=18$ armchair GNR 1 (Fig. 1a) with a width of $\sim 2.1 \mathrm{~nm}$, based on molecular modeling, surpassing all the GNRs hitherto fabricated by the bottom-up synthesis. GNR 1 features an ionization energy (IE) close to that of P3HT, making it the ideal candidate for the present study, in particular to avoid traps and energy barriers within the bi-component film. The edge decoration with long alkyl chains makes GNR 1 dispersible in common organic solvents such as tetrahydrofuran (THF), chlorobenzene

(a)

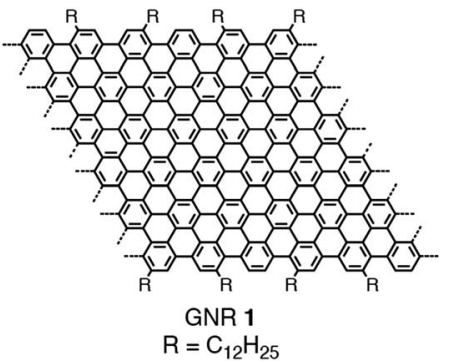

Fig. 1 Chemical formulae of (a) GNR 1 and (b) poly(3-hexylthiophene) (P3HT). and ortho-dichlorobenzene (ODCB), enabling its integration into organic electronic devices.

GNR 1 has been blended with regioregular poly(3-hexylthiophene) - P3HT (Fig. 1b). We note that the alkyl chains exposed at the peripheral positions of GNR 1 provide a good affinity for P3HT, as an attempt to minimize the occurrence of macroscopic phase segregation. This is particularly important because of the problems associated with the propensity of GNRs to form microscopic crystals that cannot be easily interfaced with metallic electrodes, therefore limiting charge injection at the metal-(semi)conductor interface. Moreover, these microcrystals are alternated by domain boundaries hampering the charge transport within the film. The effect of the GNRs at different concentrations (in the blend) on the device performance in the dark, such as the field-effect mobility and $I_{\mathrm{on}} / I_{\mathrm{off}}$, has been investigated. The influence of illumination with monochromatic light on the electrical characteristics of the device has been studied aiming at exploring the potential use of this blend as an active layer in hybrid organic phototransistors (OPTs).

\section{Results and discussion}

\section{Synthesis of GNR 1}

Scheme 1 portrays the synthetic route towards GNR 1. Our recent study has shown that the $A A$-type Yamamoto polymerization from one single monomer ${ }^{\mathbf{4 2 , 4 9 , 5 0}}$ is more efficient for the synthesis of high-molecular-weight polyphenylene precursors than the $A_{2} B_{2}$-type Suzuki polymerization that requires heterogeneous reaction conditions involving two different monomers. ${ }^{\mathbf{4 1 , 4 4 , 5 1}} A B$-type Diels-Alder polymerization has proved to be superior to transition-metal-catalyzed couplings for synthesizing long (>100 $\mathrm{nm}$ ) GNRs, but the width of GNRs is only $\sim 1$ $\mathrm{nm} .{ }^{52}$ In order to build up laterally extended, $N=18$ armchair GNR 1 by employing Yamamoto polymerization, the dihalogenated monomer 5 was designed (Scheme 1). Unlike previously
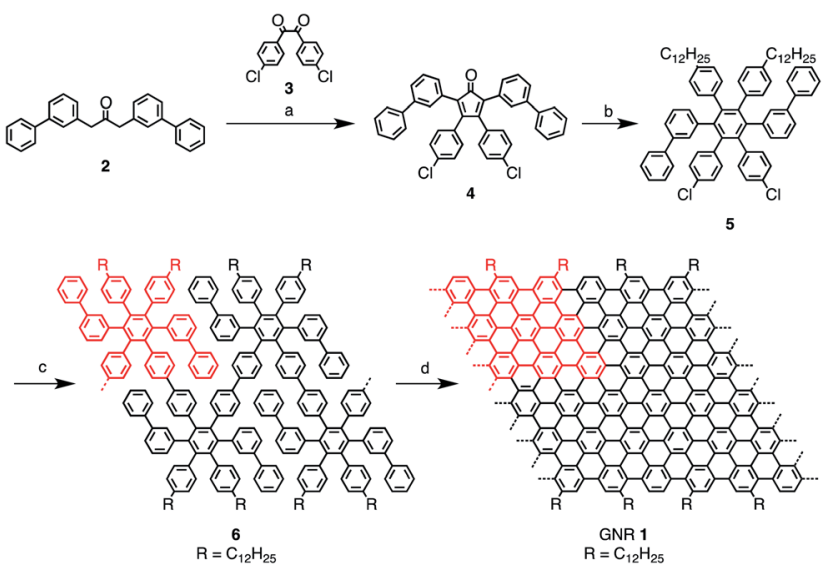

Scheme 1 Synthesis route to GNR 1. Reagents and conditions: (a) tetrabutylammonium hydroxide, $\mathrm{tBuOH}, 80{ }^{\circ} \mathrm{C}, 77 \%$; (b) bis(4-dodecylphenyl)acetylene, $\mathrm{Ph}_{2} \mathrm{O}, 230^{\circ} \mathrm{C}, \mu \mathrm{W}, 300 \mathrm{~W}, 56 \%$; (c) bis(cycloocta-(1,5)diene)nickel(0), cycloocta-(1,5)-diene, 2,2'-bipyridine, toluene/DMF, $80{ }^{\circ} \mathrm{C}, 86 \%$ (before fractionation); (d) $\mathrm{FeCl}_{3}, \mathrm{CH}_{2} \mathrm{Cl}_{2} / \mathrm{CH}_{3} \mathrm{NO}_{2}$, rt, $98 \%$. 
reported monomers that lead to GNRs with the same widths as the monomers, ${ }^{\mathbf{4 2 - 4 4 , 5 2}}$ the polymerization of monomer 5 with an $o$-terphenyl-based backbone structure would yield laterally expanded polyphenylene precursor 6 with twice the width of the monomer. Precursor 6 would be "graphitized" and "planarized" into GNR 1 with high structural definition albeit slight structural isomerization at the ends of the GNRs (Scheme S2 $\dagger$ ). For the synthesis of monomer 5, cyclopentadienone $\mathbf{4}$ was first prepared by Knoevenagel condensation of di(3-biphenylyl) acetone (2) and 4,4'-dichlorobenzil (3), and then subjected to Diels-Alder reaction with bis(4-dodecylphenyl)acetylene to yield monomer 5 (Scheme 1). Subsequently, Yamamoto polycondensation of monomer $\mathbf{5}$ was performed by employing a standard protocol, ${ }^{\mathbf{4 2 , 4 9 , 5 0}}$ followed by fractionation using recycling preparative size exclusion chromatography (SEC) to provide precursor 6 . Weight-average molecular weight $\left(M_{\mathrm{w}}\right)$ and polydispersity index (PDI) of precursor 6 were estimated by SEC analysis to be $c a .10000 \mathrm{~g} \mathrm{~mol}^{-1}$ and 1.4 , respectively, against polystyrene (PS) standards (Fig. 2a). When the poly( $p$-phenylene) (PPP) standard calibration was applied, an $M_{\mathrm{w}}$ of $c a .7300 \mathrm{~g}$ $\mathrm{mol}^{-1}$ and a PDI of 1.3 were obtained. Considering the kinked and flexible polyphenylene backbone structure of precursor 6 with long alkyl chains at the peripheral positions, the actual $M_{\mathrm{w}}$ of 6 presumably lies between these two values, i.e. 7300-10 000 $\mathrm{g} \mathrm{mol}^{-1}$. Matrix-assisted laser desorption/ionization time-offlight (MALDI-TOF) mass spectrometry (MS) analysis of precursor 6 showed a regular mass pattern extending up to $\sim 20000 \mathrm{~g} \mathrm{~mol}^{-1}$, which corresponds to the molecular weight of $\sim 20$ repeating units (Fig. $2 \mathrm{~b}$ ). Based on this result, the longitudinal length of the resulting GNR 1 could be estimated to be up to $c a .10 \mathrm{~nm}$. It should be noted however that this is most probably not the highest value, taking into account the limitation of MALDI-TOF MS for the analysis of large polymers with broad molecular weight distribution. ${ }^{\mathbf{4 1 , 4 2 , 5 3}}$

The intramolecular cyclodehydrogenation of precursor 6 was carried out by using an established method, applying iron(III) chloride as the Lewis acid and oxidant in a mixture of dichloromethane and nitromethane. ${ }^{\mathbf{4 1 4 4 , 4 7}}$ MALDI-TOF MS analysis of GNR 1 in linear mode displayed a regular mass pattern similar to that of precursor 6 (Fig. S4 in the ESI $\dagger$ ). Although the peaks were strongly broadened, the interval was roughly $\sim 1000 \mathrm{~g} \mathrm{~mol}^{-1}$, corresponding to the molecular weight of one repeating unit of GNR 1, i.e. $991 \mathrm{~g} \mathrm{~mol}^{-1}$. More accurate analysis as well as detection of mass exceeding $15000 \mathrm{~g} \mathrm{~mol}^{-1}$ was not possible most probably due to the strong self-association tendency of larger GNRs and fragmentations at higher laser powers. ${ }^{42,53}$

Comparison of Fourier transform infrared (FTIR) spectra of precursor 6 and GNR 1 displayed disappearance of the band from the rotation of free phenyl rings at $4052 \mathrm{~cm}^{-1}$, as well as strong attenuation of the signal triad from aromatic $\mathrm{C}-\mathrm{H}$ stretching vibrations at 3029,3054 , and $3084 \mathrm{~cm}^{-1}$, after the cyclodehydrogenation (Fig. 3). ${ }^{\mathbf{4 1 , 4 2 , 5 4}}$ Further, out-of-plane (opla) $\mathrm{C}-\mathrm{H}$ deformation bands in the fingerprint regions such as at 698,841 , and $897 \mathrm{~cm}^{-1}$ vanished, validating the disappearance of mono- and di-substituted benzene rings. ${ }^{54,55}$ These results indicated the high efficiency of the cyclodehydrogenation of precursor 6 leading to successful formation of GNR 1.

To further characterize the cyclodehydrogenation of precursor 6 into GNR 1, we have used liquid- and solid-state ${ }^{1} \mathrm{H}$ NMR spectroscopy as summarized in Fig. 4. This analysis makes it possible to follow the structural changes before and after cyclodehydrogenation, since the ${ }^{1} \mathrm{H}$ chemical shift (position and line width) is known to be a highly sensitive probe for non-
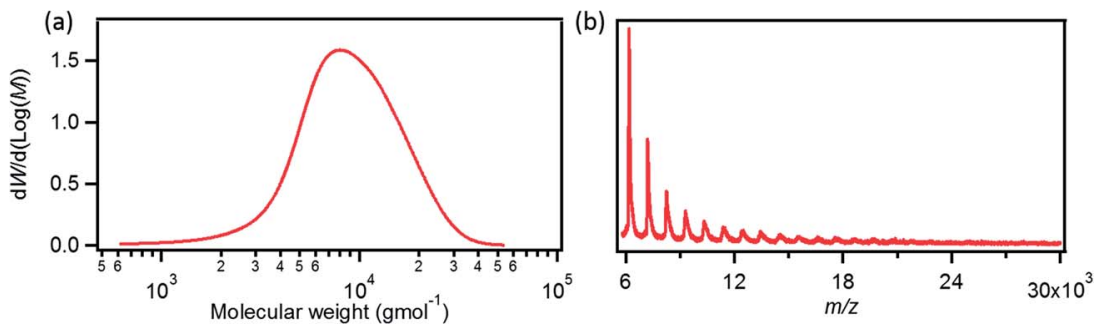

Fig. 2 (a) Molecular weight distribution of polyphenylene precursor 6 (SEC analysis, eluent: THF, polystyrene standard). (b) Linear-mode MALDITOF MS spectrum of polyphenylene precursor 6 .

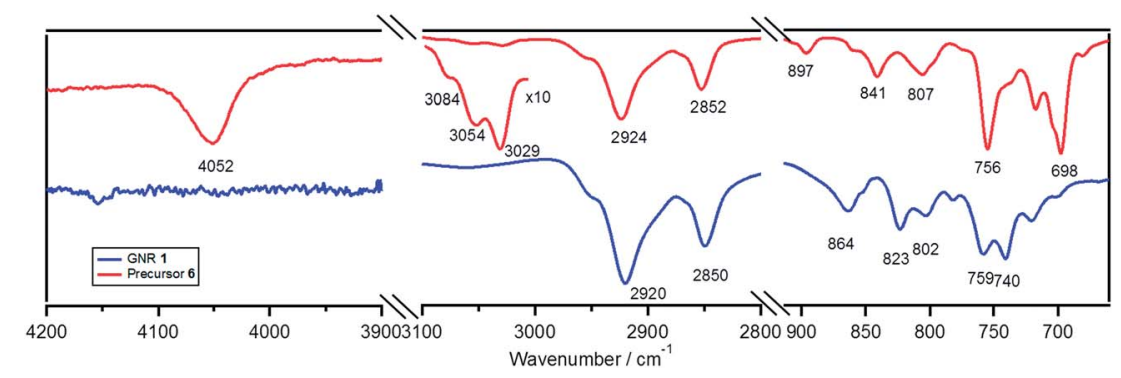

Fig. 3 Representative FTIR spectral regions of polyphenylene precursor 6 (red) and GNR 1 (blue). 
covalent interactions. ${ }^{56-59}$ The ${ }^{1} \mathrm{H}$ NMR spectra in Fig. $4 \mathrm{a}$ and b unambiguously reveal that precursor 6 possesses a semi-flexible/semi-rigid structure due to the fact that the overall ${ }^{1} \mathrm{H}$ line width from the polyphenylene groups at $\sim 7.0 \mathrm{ppm}$ is almost unchanged, when going from the liquid- to the solid-state NMR spectrum (Fig. $4 \mathrm{a}$ and b, respectively). After cyclodehydrogenation, the ${ }^{1} \mathrm{H}$ magic-angle spinning (MAS) NMR spectrum of GNR 1 shown in Fig. 4c includes significantly broadened ${ }^{1} \mathrm{H}$ resonances, where, in particular, those related to the aromatic signals are shifted to a higher frequency (or equivalently to lower field). The broad nature and shift of the aromatic ${ }^{1} \mathrm{H}$ signals demonstrate that these are part of an extended $\pi$-conjugated system, ${ }^{\mathbf{6 0 , 6 1}}$ and that GNR $\mathbf{1}$ behaves like a rigid solid, consistent with the formation of GNRs. Fig. $4 \mathrm{~d}$ and e display the $2 \mathrm{~d}^{1} \mathrm{H}^{-1} \mathrm{H}$ double quantum-single quantum (DQSQ) NMR correlation experiments mapping out the spatial connectivities between the different ${ }^{1} \mathrm{H}$ signals for precursor 6 and GNR 1. ${ }^{62,63}$ For precursor 6, the $2 \mathrm{D}^{1} \mathrm{H}^{1}{ }^{1} \mathrm{H}$ DQ-SQ spectrum includes narrow ${ }^{1} \mathrm{H}-{ }^{1} \mathrm{H}$ correlation signals between polyphenylene groups as well as between the polyphenylene groups and the dodecyl side chains as one might expect for a flexible structure. However, after cyclodehydrogenation, GNR 1 shows a broad, stretched split ridge of ${ }^{1} \mathrm{H}-{ }^{1} \mathrm{H}$ correlation signals close to the spectrum diagonal in the range between $\sim 9$ and $12 \mathrm{ppm}$ (Fig. 4e), in addition to a clear, albeit broad, ${ }^{1} \mathrm{H}-{ }^{1} \mathrm{H}$ correlation between aromatic protons at $\sim 6-9 \mathrm{ppm}$ and those of the

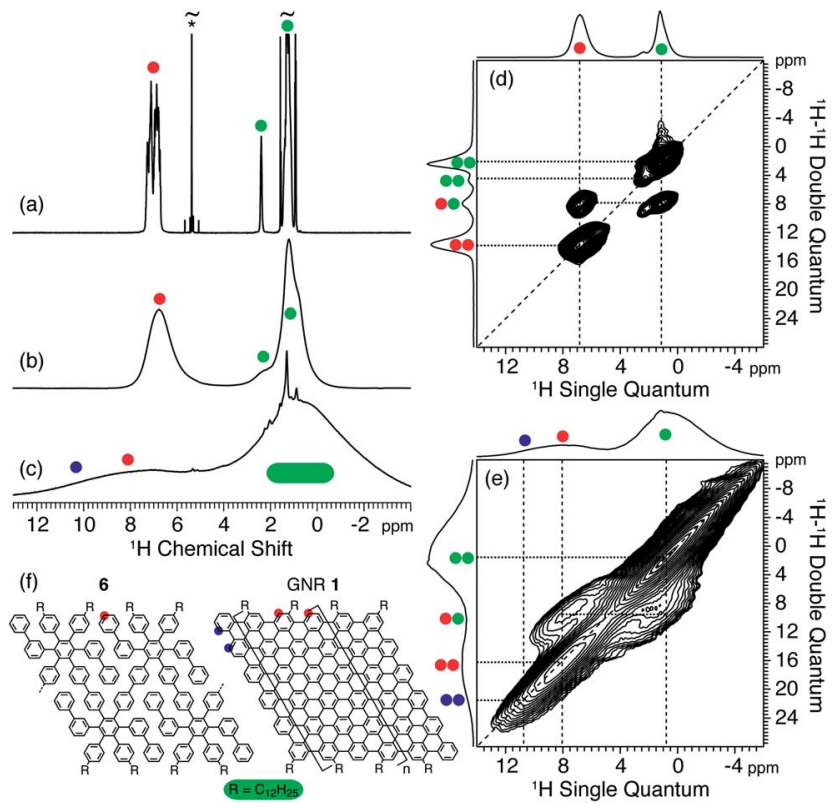

Fig. 4 Liquid- and solid-state ${ }^{1} \mathrm{H}$ NMR spectra of (a) precursor 6 in $\mathrm{CD}_{2} \mathrm{Cl}_{2}$, and (b and $\mathrm{c}$ ) solid samples of precursor 6 and GNR 1, respectively. The $2 \mathrm{~d}^{1} \mathrm{H}-{ }^{1} \mathrm{H} D Q-S Q$ correlation spectra of (d) precursor 6 and (e) GNR 1 were recorded using two rotor periods of DQ recoupling. The ${ }^{1} \mathrm{H}$ signals are assigned using the color code given in (f); all the aromatic signals of 6 are represented by the red color. The liquid-state spectrum in (a) was recorded at 7.05 $\mathrm{T}\left(300.13 \mathrm{MHz}\right.$ for $\left.{ }^{1} \mathrm{H}\right)$, while the solid-state NMR experiments in $(b-e)$ were obtained at 16.45 $\mathrm{T}\left(700.21 \mathrm{MHz}\right.$ for $\left.{ }^{1} \mathrm{H}\right)$ using a MAS frequency of $59524 \mathrm{~Hz}$. The asterisks in (a) mark the residual proton signals of $\mathrm{CD}_{2} \mathrm{Cl}_{2}$. The sharp signals in (c) represent residual solvent present in the sample. dodecyl side chains. Based on these spectral differences we can make the assignment shown in Fig. 4f, where the highfrequency part is attributed to the protons at the end of GNR 1 (blue) and the lower-frequency part to the protons of the edge (red) in close spatial proximity to the dodecyl side chains. Note that the edge protons (red) of GNR 1 only show a small shift of 1-2 ppm to high frequency when compared to precursor 6 , while a much larger shift of $\sim 2-5 \mathrm{ppm}$ is observed for the end protons (blue). These differences illustrate that the bulk sample of GNR 1 is heterogeneously packed, shifting the ${ }^{1} \mathrm{H}$ signals as a result of the different magnitudes for the aromatic ring currents of stacked GNRs. ${ }^{64,65}$

The successful conversion of precursor 6 into GNR 1 was further verified by investigation of a model monomer S4, for which the efficient cyclodehydrogenation was demonstrated by the combination of ${ }^{1} \mathrm{H}$ NMR and MALDI-TOF MS analysis (see $\mathrm{ESI} \dagger$ ).

GNR 1 can be easily dispersed in common organic solvents such as THF, chlorobenzene, and ODCB by applying mild sonication to give brown dispersions. Such dispersions of GNR 1 with typical concentrations up to $\sim 1 \mathrm{mg} \mathrm{mL}^{-1}$ in ODCB are stable for at least one week without apparent precipitation. The UV-vis absorption spectrum of a dispersion of GNR 1 in ODCB shows an absorption maximum at $410 \mathrm{~nm}$ with the absorption edge at $\sim 800 \mathrm{~nm}$, corresponding to an optical bandgap $\sim 1.6 \mathrm{eV}$ (Fig. S5 $\dagger$ ). Subsequently, different volumes of such dispersion in ODCB are mixed with solutions of P3HT for the fabrication of GNR-P3HT blend films with various GNR loading ratios by codeposition.

\section{Electrical, structural and morphological characteristics in the dark}

Ambient photoelectron spectroscopy (PS) measurements of the pristine GNR 1 dropcasted film indicate a HOMO level of 5.010 $\pm 0.063 \mathrm{eV}$, close to that of P3HT, i.e. $4.860 \pm 0.015 \mathrm{eV}$. Thereby, this result makes GNR 1 an ideal candidate for our case study.

Then we fabricated devices at various GNR loading ratios $(0$, 2, 5, 11 and $24 \mathrm{wt} \%$ with respect to P3HT), having different channel lengths $(L)$ ranging from $60 \mu \mathrm{m}$ to $120 \mu \mathrm{m}$. Fig. 5a displays transfer characteristics of a $120 \mu \mathrm{m}$ channel length device with $24 \%$ GNRs with its output characteristics shown as the inset, the device exhibits a typical $p$-type behavior. The extracted hole mobility (from the saturation regime at $V_{\mathrm{D}}=-60$ $\mathrm{V})$, threshold voltage $\left(V_{\mathrm{th}}\right)$ and $I_{\mathrm{on}} / I_{\text {off }}$ is $2.8 \times 10^{-2} \mathrm{~cm}^{2} \mathrm{~V}^{-1} \mathrm{~s}^{-1}$, $-8.9 \mathrm{~V}$ and $\sim 10^{3}$ respectively. The same channel length device based on pristine P3HT was also studied (transfer and output characteristics are shown in Fig. S6 in the ESI $\dagger$ ). The only parameter that exhibited a notable change is the field-effect mobility $\mu$, which amounted to $7.3 \times 10^{-3} \mathrm{~cm}^{2} \mathrm{~V}^{-1} \mathrm{~s}^{-1}$. This shows that the performance of the device is enhanced in the presence of GNRs. Such an improvement can be ascribed to (i) the percolation pathway for the charges provided by the GNRs, or (ii) the ionization energy of the $24 \%$ blend, as determined by ambient PS, is $4.790 \pm 0.016 \mathrm{eV}$ (i.e. lowered by $0.07 \mathrm{eV}$ as compared to the pure P3HT $4.860 \pm 0.015 \mathrm{eV}$ ), thus it better matches the work function of the Au electrodes $(4.810 \pm 0.020$ 
(a) 10

蛴 1 -
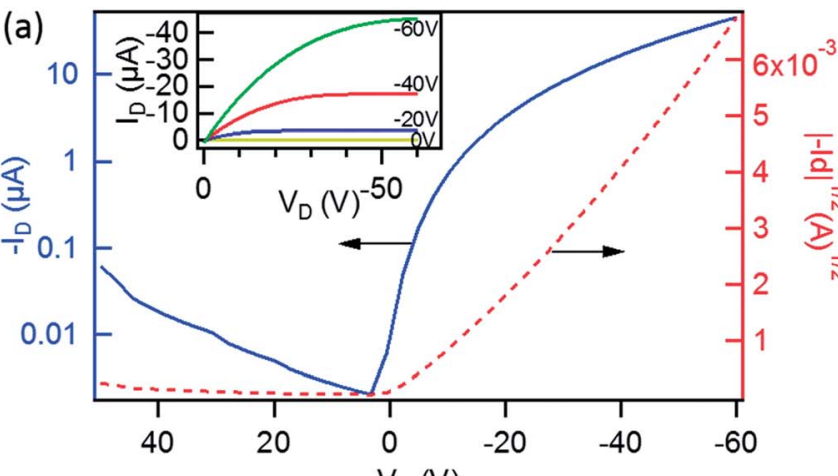

(c)

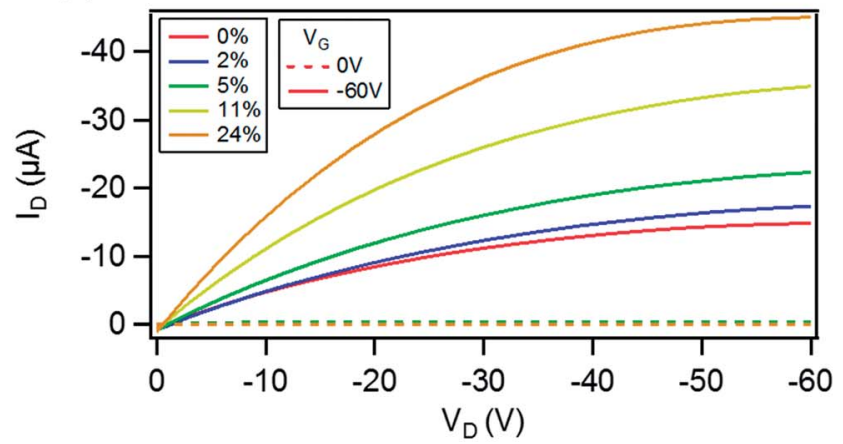

(b)

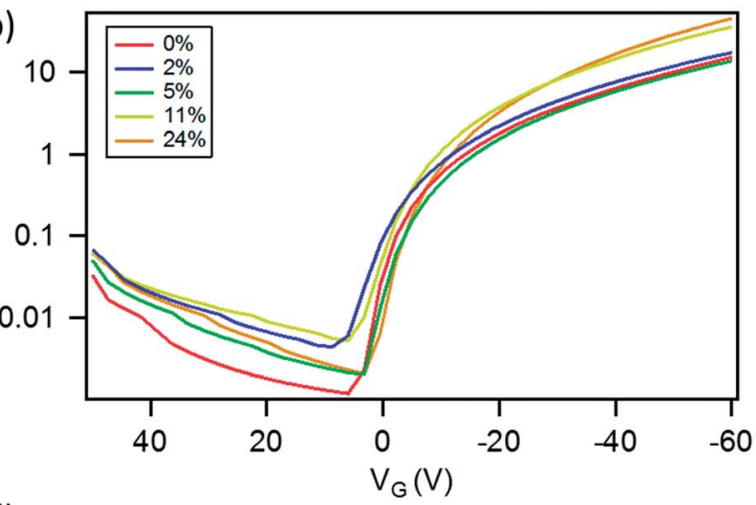

(d)

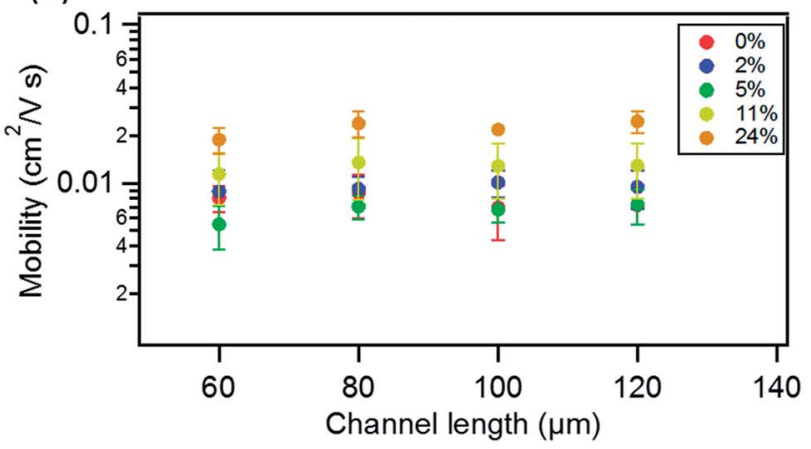

Fig. 5 (a) Transfer characteristics of bottom-gate bottom-contact devices ( $L=120 \mu \mathrm{m}$ ) prepared by blending $24 \%$ of GNRs with P3HT (left axis in the log scale). The inset shows their output characteristics. (b) Transfer characteristics of bottom-gate bottom-contact devices $(L=120 \mu \mathrm{m})$ prepared by different blend percentages of GNRs with P3HT, and (c) their output characteristics (solid line at $V_{G}=-60 \mathrm{~V}$ and dashed line at $V_{G}=$ $0 \mathrm{~V}$; the rest of data at $V_{\mathrm{G}}=-20 \mathrm{~V}$ and $-40 \mathrm{~V}$ have been omitted for clarity). (d) Variation of the field-effect mobility (left axis in the log scale) with the channel length $(60,80,100$ and $120 \mu \mathrm{m})$ at different blend percentages of GNRs with respect to P3HT.

eV) enabling a better charge injection. A comparison of the transfer characteristics (at $V_{\mathrm{D}}=-60 \mathrm{~V}$ ) of the devices with the same $L=120 \mu \mathrm{m}$ at different concentrations of GNRs is shown in Fig. 5b. It reveals an increase in $I_{\mathrm{d}}$ with increase in the relative amount of GNRs up to a factor of 3 when comparing the $24 \%$ blend with the pristine devices ( $0 \%$ blend). Such a trend is also evident in Fig. $5 \mathrm{c}$ which compares the output characteristics of the same blend devices at $V_{\mathrm{G}}=0 \mathrm{~V}$ (dashed line) and at $V_{\mathrm{G}}=$ $-60 \mathrm{~V}$ (solid line).

Fig. 5d (see also Fig. S7 in the ESI $†$ ) shows the high variation of hole field-effect mobility at different concentrations of GNRs and for devices with different $L$. In particular, at 2 and $5 \%$ loadings, the field-effect mobility is comparable to that of the devices with pristine P3HT. The mobility increased $\sim 1.7$ times when the content of GNRs augments to $11 \%$, and by a factor of 3 at $24 \%$ with respect to P3HT. The enhanced performance can be attributed to the above mentioned reasons, and mainly to the amount of GNRs that can facilitate the charge transport within the conduction channel, as observed in similar studies on the P3HT blend with graphene, ${ }^{26}$ or with functionalized carbon nanotubes. ${ }^{29}$ Enhanced performances were also observed for other p-type polymers printed on the top of a graphene layer inside the channel. ${ }^{66}$ In fact, it has been demonstrated that when P3HT is blended with another semiconductor, there is a critical concentration for the enhancement of the mobility. ${ }^{28,67}$ When comparing all the devices at different blend \% and different $L$ spanning from $60 \mu \mathrm{m}$ to $120 \mu \mathrm{m}$, the average $V_{\text {th }}$ was found to range between 2 and $-5 \mathrm{~V}$, and to be slightly higher (up to $-8 \mathrm{~V}$ ) for those with $24 \%$ of GNR 1 . Significantly, the average $I_{\text {on }} / I_{\text {off }}$ ranged between $10^{3}$ and $10^{4}$, and appeared unaffected by the presence of GNRs (see Fig. S8 in the ESI†), unlike blends with graphene where the $I_{\mathrm{on}} / I_{\text {off }}$ markedly decreases at higher concentrations..$^{26}$ Electrical characterization was also performed on shorter channel lengths ranging from $2.5 \mu \mathrm{m}$ to $20 \mu \mathrm{m}$, with the results being reported in Fig. S9 in the ESI. $\dagger$

To gain further insight into the relation between electrical properties and structure within the blend films, Grazing Incidence X-ray Diffraction (GIXD) measurements were carried out on the spin-coated films. This reveals that the addition of GNR 1 strongly influences the order within P3HT films. In the pattern recorded for the monocomponent P3HT film (Fig. 6a) the $h 00$ series, corresponding to the interchain distance measured in the plane of aromatic rings, is discernible up to the third diffraction order. Another characteristic peak, originating from the $\pi-\pi$ stacking of P3HT, is visible on the equator, indicating that in the pure P3HT film the macromolecules adopt an edgeon orientation in the crystalline domains. The chain-to-chain distance (16.4 $\AA$ ), the $\pi$-stacking distance (3.6 $\AA$ ) as well as the approximated coherence length of the $\pi-\pi$ stacking derived from GIXD data ( $\sim 70 \AA$; Fig. 6a and c) are typical for P3HT films. ${ }^{68-70}$ The addition of GNR to P3HT affects the arrangement of the crystalline structure of the polymer. The absence of 

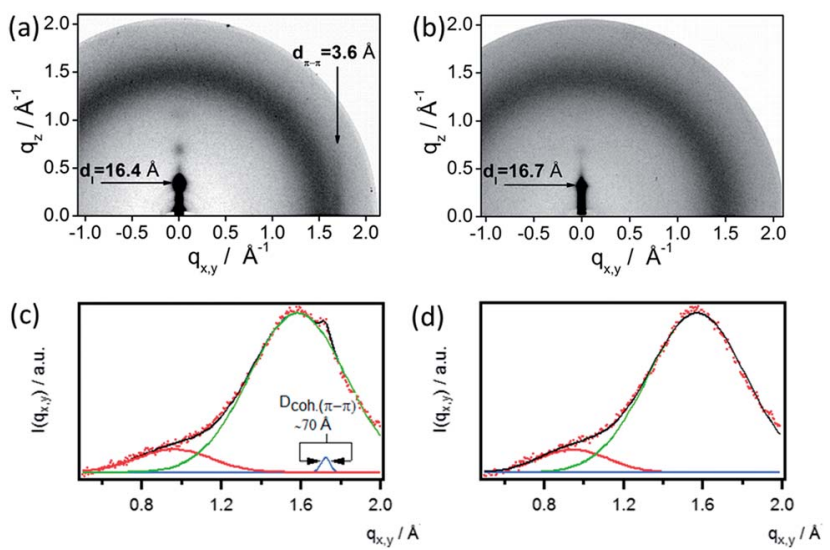

Fig. 6 GIXD patterns of (a) P3HT film, (b) P3HT $+2 w t \%$ GNR, and the corresponding horizontal integrations of (c) P3HT and (d) the blend.

characteristic equatorial reflections at $q_{x, y} \approx 1.72 \AA^{-1}$ related to the $\pi$-stacking reflection in XRD patterns of all the blends (Fig. 6b and d) indicates that even a small amount of GNRs, such as $2 \mathrm{wt} \%$, hinders the formation of coherent $\pi$-stacks. Increasing amounts of GNRs cause also a gradual decrease in the integral intensity of the 100 reflection (at $q_{z}=0.377 \AA^{-1}$ ), providing evidence for a decrease in lamellar ordering of $\mathrm{P} 3 \mathrm{HT}$ macromolecules, hence in the overall crystallinity of the polymer. Unfortunately, a strong diffuse scattering of X-rays on the GNRs overlaps with P3HT peaks, which does not permit a quantitative analysis of the polymer crystallinity. On the other hand, such a strong scattering suggests the existence of a large interface area between the GNRs and P3HT. Increasing the GNR content beyond $5 \mathrm{wt} \%$ causes almost complete disappearance of diffraction features from the crystalline fraction of P3HT, which indicates that at the higher content of GNRs no significant polymer crystals are formed. We suggest that the increase of the mobility in the blend can be attributed to the presence of
GNRs and aggregates thereof, providing favorable pathways for the transport of charges counteracting the effect of decreased crystallinity in the P3HT domains upon addition of GNR.

As the $q$-range in the GIXD experiments allowed studying structures with length scales up to a very few nanometers, in order to analyze the occurrence of possible phase separation on a length scale ranging from a few nanometers up to a few tens of micrometers we used atomic force microscopy in the tapping mode (TM-AFM). The pristine P3HT film from ODCB solution is homogeneous featuring fibrillar and grain-like structures on the tens of nanometers scale (Fig. 7a). At $24 \%$, the P3HT/GNR films are inhomogeneous due to the aggregation of the GNRs as shown in Fig. 7b. These aggregates having a height ranging from 60 to $200 \mathrm{~nm}$ and a width spanning from 0.6 to $2 \mu \mathrm{m}$, were found to be randomly distributed within the polymer matrix. Their frequency of occurrence inside the channel, i.e. the number of aggregates located in the source-drain gap, was proportional to the concentration of GNRs; conversely, the aggregate size was found independent of the concentration (see Fig. S11 in the ESI†). This clarifies how the aggregates of GNRs, and not single GNRs, act as an "electronic bridge" between P3HT domains. Because of the high propensity of our GNRs to undergo aggregation when deposited on a solid surface, single component polycrystalline structures are obtained which are difficult to characterize, in line with observations on similar liquid phase-processable GNRs. ${ }^{52}$ It is indeed surprising to see the mild increase in field-effect mobility upon blending P3HT with the GNRs, given that it is known that a $2 \mathrm{~nm}$ wide GNR can exhibit a mobility around $100 \mathrm{~cm}^{2} \mathrm{~V}^{-1} \mathrm{~s}^{-1} .^{71}$ Yet, a maximum field-effect mobility of $3.25 \times 10^{-2} \mathrm{~cm}^{2} \mathrm{~V}^{-1} \mathrm{~s}^{-1}$ was observed for a similar arm-chair GNR deposited from ODCB. ${ }^{72}$ Consequently, the reason for the limited increase in mobility is likely the simultaneous effect of decreased crystallinity with the P3HT film upon blending and charge scattering when encountering GNR aggregates.

\section{(a)}

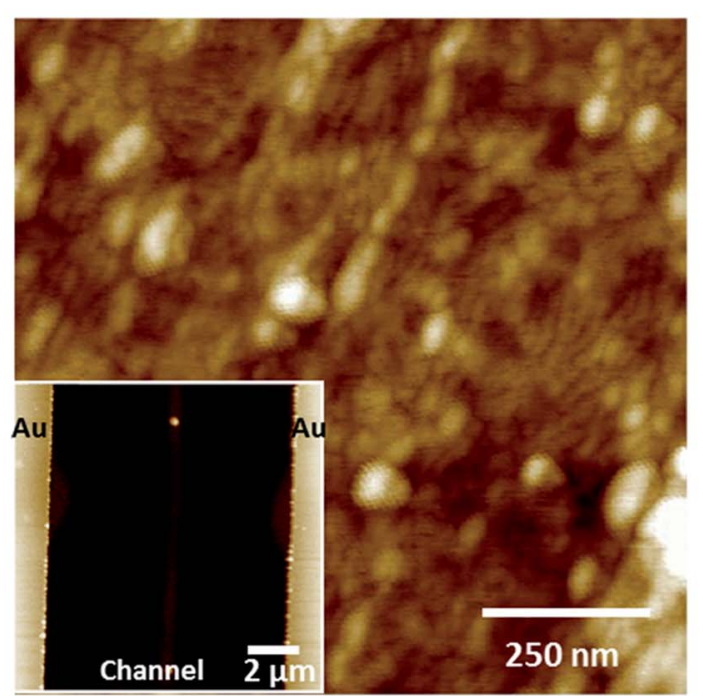

(b)

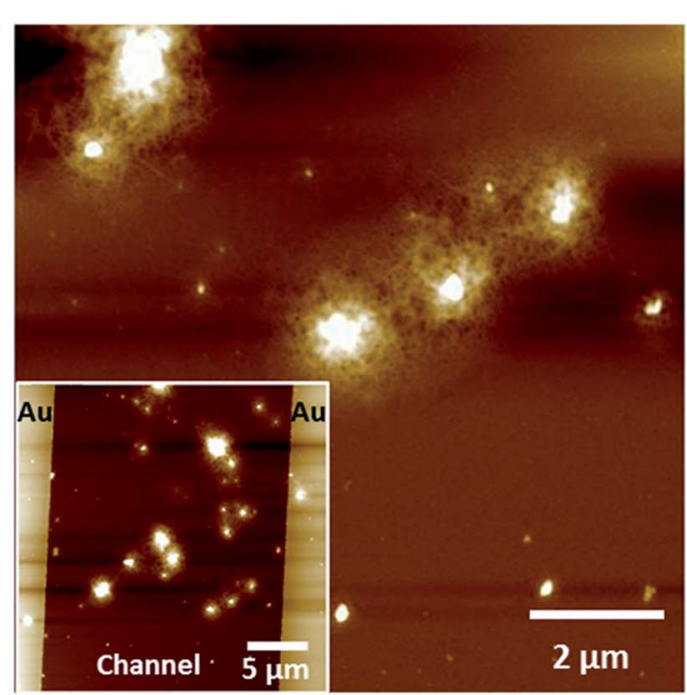

Fig. 7 AFM images scanned inside the channel of films of: (a) pristine P3HT and (b) 24\% GNR with P3HT. The insets show the scan at a larger scale. Z-scales: (a) $17 \mathrm{~nm}$, (b) $75 \mathrm{~nm}$, inset (a) $47 \mathrm{~nm}$, inset (b) $92 \mathrm{~nm}$. 
(a)

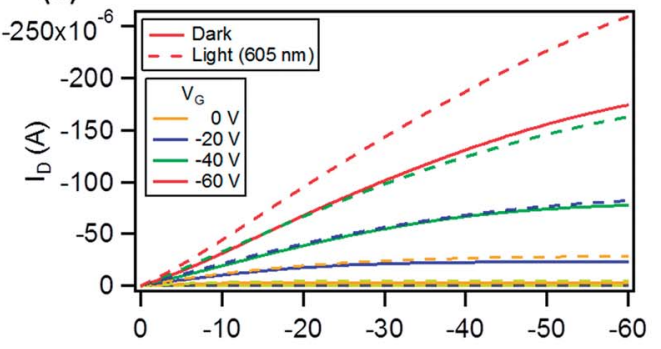

(c)

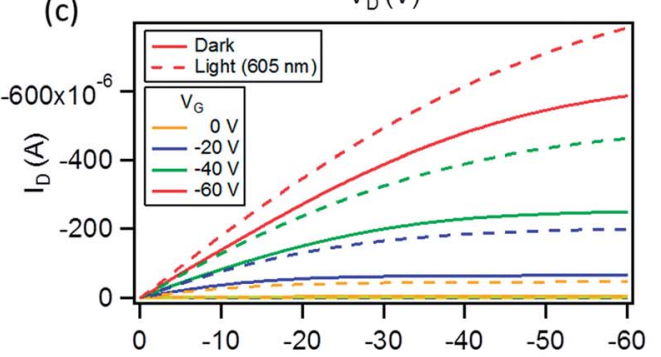

(e)

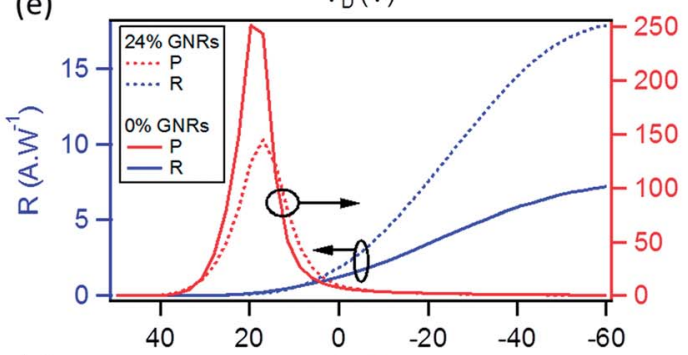

(g) $\quad v_{G}(\mathrm{~V})$

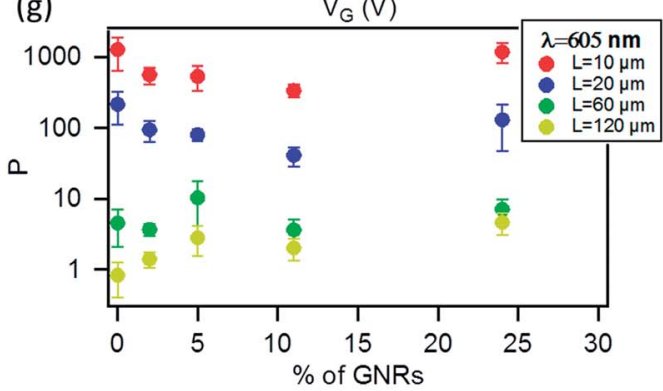

(b)
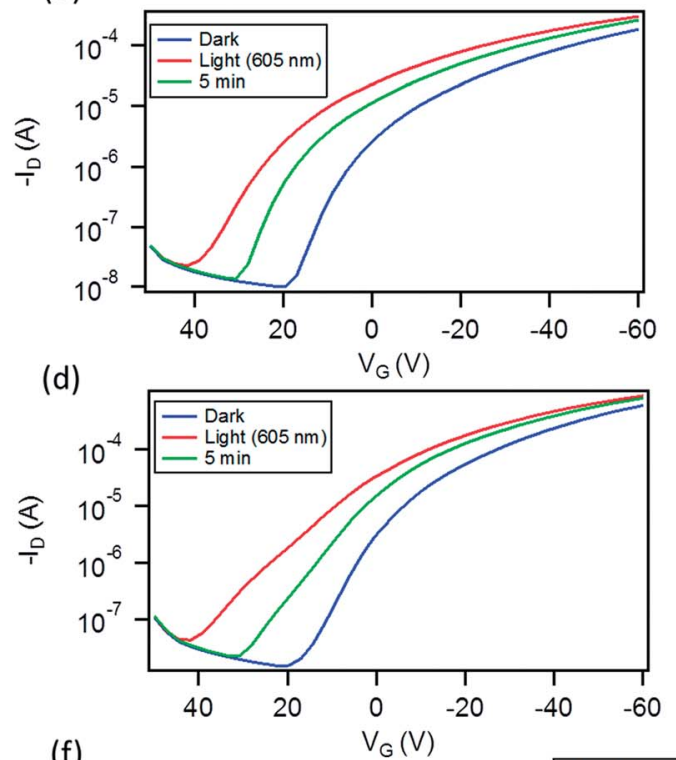

(f)

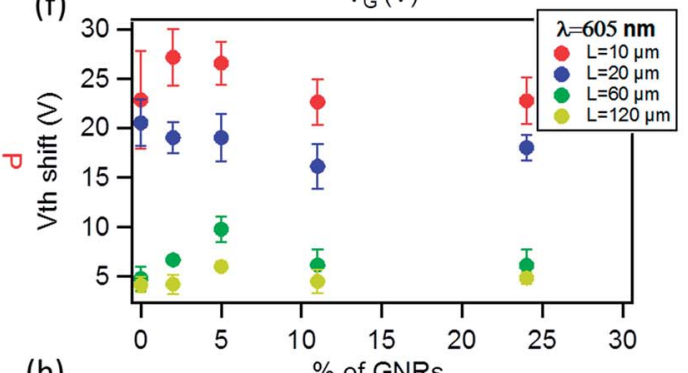

(h)

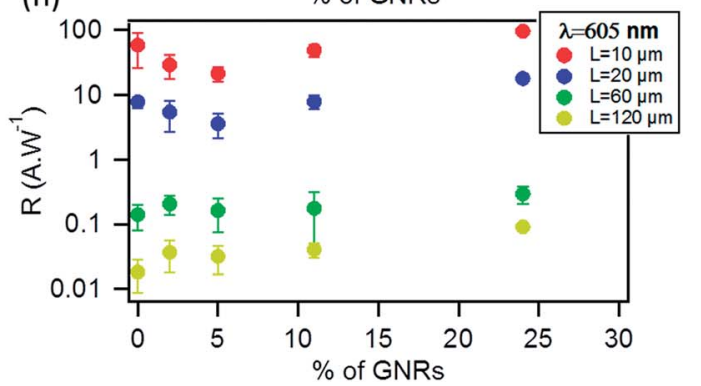

Fig. 8 Comparison of (a) output and (b) transfer characteristics at $V_{D}=-60 \mathrm{~V}$ of pristine P3HT OFET; (c) output and (d) transfer characteristics at $V_{D}=-60 \mathrm{~V}$ of a device with $24 \%$ GNR blend with P3HT with the same channel length $L=20 \mu \mathrm{m}$ measured in the dark and under monochromatic light $\left(\lambda=605 \mathrm{~nm} ; 8.17 \mathrm{~mW} \mathrm{~cm}^{-2}\right.$ ). (e) Variation of the responsivity $R$ (blue curves) and photosensitivity $P$ (red curves) with $V_{\mathrm{G}}$ at $V_{\mathrm{D}}=-60 \mathrm{~V}$ at $\lambda=$ $605 \mathrm{~nm}$ for OFETs ( $L=20 \mu \mathrm{m}$ ) with $0 \%$ (solid lines) and 24\% blends of GNRs (dashed lines). (f) Threshold voltage shift under illumination with monochromatic light $\left(\lambda=605 \mathrm{~nm} ; 8.17 \mathrm{~mW} \mathrm{~cm}^{-2}\right)$ for 4 channel length $(L=10,20,60$ and $120 \mu \mathrm{m})$ OFETs based on different blend percentages of GNRs with respect to P3HT. (g) Photosensivity (extracted at $V_{G}=20 \mathrm{~V}$ ), and (h) responsivity of these devices (extracted at $V_{G}=-60 \mathrm{~V}$ ) both at $V_{D}=-60 \mathrm{~V}$. The left axis is expressed in log-scale.

\section{Electrical characteristics under illumination}

Measurements of the photoresponse under illumination at 605 $\mathrm{nm}$ and $560 \mathrm{~nm}$ were carried out to investigate both the potential application of these OFETs in optoelectronics and the influence of the GNRs on the photo-generated carriers. The two wavelengths were chosen because they correspond to two absorption peaks of the films (see Fig. S12 in the ESI $\dagger$ ).

The difference in output $\left(I_{\mathrm{D}}-V_{\mathrm{D}}\right)$ and transfer $\left(I_{\mathrm{D}}-V_{\mathrm{G}}\right)$ characteristics of a $0 \%$ and $24 \%$ OFET $(L=20 \mu \mathrm{m})$ in the dark and under illumination with monochromatic light $\left(8.17 \mathrm{~mW} \mathrm{~cm}^{-2}\right)$ at $\lambda=605 \mathrm{~nm}$ is illustrated in Fig. 8a-d. The electrical characteristics of a pristine $\mathrm{P} 3 \mathrm{HT}$ based device, i.e. with $0 \%$ GNR in the blend, is shown in Fig. 8a and b. It displays an increase of the drain current in the $I_{\mathrm{D}}-V_{\mathrm{D}}$ and $I_{\mathrm{D}}-V_{\mathrm{G}}$ curves upon irradiation with monochromatic light as a result of the photogeneration of excitons that dissociate into free charge carriers which subsequently move towards the electrode under the influence of the electric field. ${ }^{73}$ The same was observed for the $24 \%$ blend. The only difference is that at $V_{\mathrm{G}}=20 \mathrm{~V}\left(V_{\mathrm{D}}=-60 \mathrm{~V}\right)$ the ratio of the photocurrent $\left(I_{\mathrm{ph}}\right)$ (calculated by subtracting the dark current $\left(I_{\mathrm{dark}}\right)$ from the current under light $\left(I_{\text {light }}\right.$, i.e. $I_{\mathrm{ph}}=$ $I_{\text {light }}-I_{\text {dark }}$ ) to $I_{\text {dark }}$ is 251.71 for the device with $0 \%$ GNR (see 
Fig. 8b, e) whereas it was much lower (145.1) for the device with $24 \%$ blend (see Fig. 8d, e). This ratio, called photosensitivity $P$ :

$$
P=\frac{I_{\text {light }}-I_{\text {dark }}}{I_{\text {dark }}}
$$

is one of the key figures of merit of an organic phototransistor. Another important parameter is the responsivity $R$ :

$$
R=\frac{I_{\text {light }}-I_{\text {dark }}}{E A}
$$

where $E$ is the irradiance of the incident light and $A$ is the effective device area. In contrast to $P, R$ values $\left(18 \mathrm{~A} \mathrm{~W}^{-1}\right)$ of the device with $24 \%$ blend (Fig. $8 \mathrm{e}$ blue plot with dashed line) is more than twice that of the $0 \%$ blend $\left(7.2 \mathrm{~A} \mathrm{~W}^{-1}\right)$ at $V_{\mathrm{G}}=-60 \mathrm{~V}$ $\left(V_{\mathrm{D}}=-60 \mathrm{~V}\right)$ (Fig. 7e blue plot with solid line). Considering eqn (2) and since $E$ and $A$ are equal for both devices, the higher $R$ in the $24 \%$ blend indicates that $I_{\mathrm{ph}}$ is higher than that of the $0 \%$. This might be due to (i) the presence of more carriers resulting from a better dissociation of excitons in the presence of GNRs, and/or (ii) the GNRs at this concentration helping conducting more charges, hence collecting more photogenerated ones at the electrodes. To assess the validity of these propositions, OFETs with different channel lengths $(L=10,20,60$ and 120 $\mu \mathrm{m})$ and different loads of GNRs with respect to $\mathrm{P} 3 \mathrm{HT}(0 \%, 2 \%$, $5 \%, 11 \%$ and $24 \%$ ) were studied at this wavelength. We observed the highest $R=94.46 \mathrm{~A} \mathrm{~W}^{-1}$ and $P=1463.26$ for the $24 \%$ device for the $10 \mu \mathrm{m}$ channel length. The photoresponse decreases by several orders of magnitude with increasing channel length (see Fig. 8f, $g$ and h). This is in line with the previous study done on perylenebis(dicarboximide)s (PDIs) based organic phototransistors. ${ }^{74}$ When considering the devices with $L=10 \mu \mathrm{m}$ at different blend percentages, under illumination, the average threshold voltage shift was in the same range, $c a$. $23 \mathrm{~V}$, for $0 \%, 11 \%$, and $24 \%$ blends. However, it was slightly higher, ca. $27 \mathrm{~V}$, for $2 \%$ and $5 \%$ blends. A similar trend was observed for all the channel lengths except for $L=20$ $\mu \mathrm{m}$ devices (see Fig. 8f). The origin of the threshold voltage shift was attributed to (i) a reduction in the energy level bending resulting from the Fermi level which moves close to the highest occupied molecular orbital under illumination ${ }^{75}$ and (ii) the change in the trap states of the semiconductor. ${ }^{76-79}$ It was reported that the apparent shift comes from the increase in the total current because of the photo-generated current. ${ }^{80}$ In our case, under illumination, one cannot detect any trend in the $V_{\text {th }}$ shift with increasing GNR percentage and channel length, probably due to the random distribution of the GNR aggregates inside the channel and the difference in their size as shown by AFM images. On one hand, in OFETs with $L=10$ and $20 \mu \mathrm{m}$, the average $P$ (Fig. 8g) decreases with increasing amount of GNRs and is more significant at $2 \%$ and $5 \%$ (as compared to $0 \%$ ) unlike the devices with $L=60$ and $120 \mu \mathrm{m}$. On the other hand, $R$ for $L=10$ and $20 \mu \mathrm{m}$ (Fig. 8h) decreases with increasing GNR \% in the blend except for $24 \%$, where it is higher than the $0 \%$ blend by a factor of two, approximately similar to the devices with $L=60$ and $120 \mu \mathrm{m}$; however, in the latter case $R$ increases with the blend percentage. This demonstrates that the ability of a device (with short $L$ ) to convert light into electric current is three times larger in the presence of $24 \%$ GNRs.

The photoresponse was also measured at $\lambda=560 \mathrm{~nm}(9.44$ $\mathrm{mW} \mathrm{cm}{ }^{-2}$ ), corresponding to the maximum absorption of the P3HT films, as shown in E12 in the ESI. $\uparrow$ A much lower photoresponse is seen for $L=60$ and $120 \mu \mathrm{m}$. By comparing the trend with the GNR\% for the responsivity and photosensitivity (a)

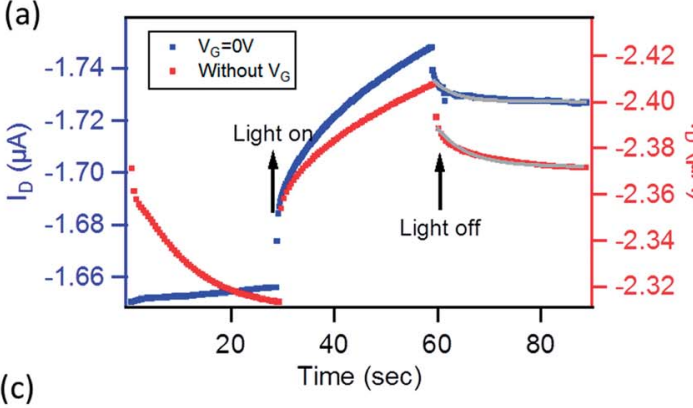

(c)

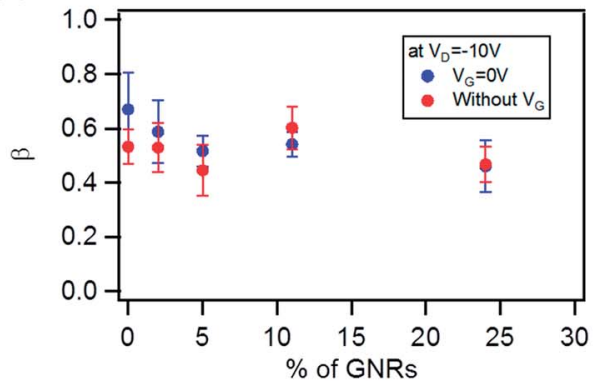

(b)

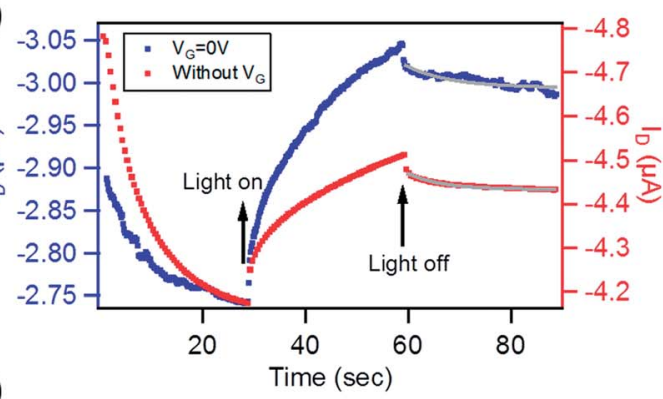

(d)

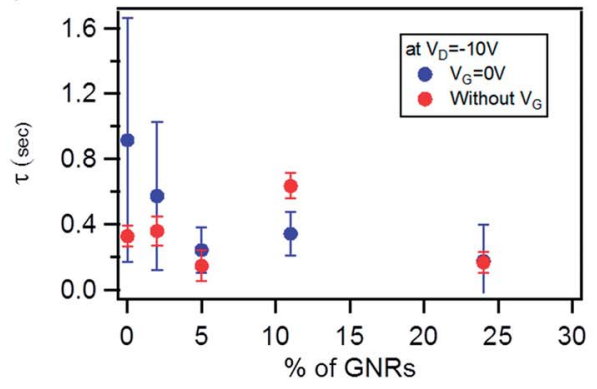

Fig. 9 (a) Relaxation characteristics of the photoinduced current after irradiation with monochromatic light $\left(\lambda=560 \mathrm{~nm}, 9.44 \mathrm{~mW} \mathrm{~cm}^{-2}\right)$ (light on at $30 \mathrm{~s}$, light off at $60 \mathrm{~s}$ ) for a $0 \%$ blend, and (b) $24 \%$ GNR blend P3HT. The gray line is the fitting by Kohlrausch's law. Average variation of: (c) the exponent $\beta$ (indicating the degree of the disorder of the material), and (d) the relaxation lifetime $\tau$ at different blend percentages (done on 5 to 6 devices for each blend \%). 
(a)

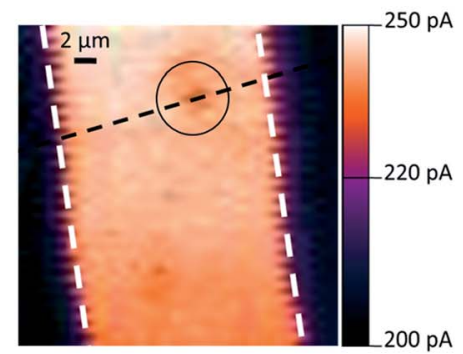

(b)

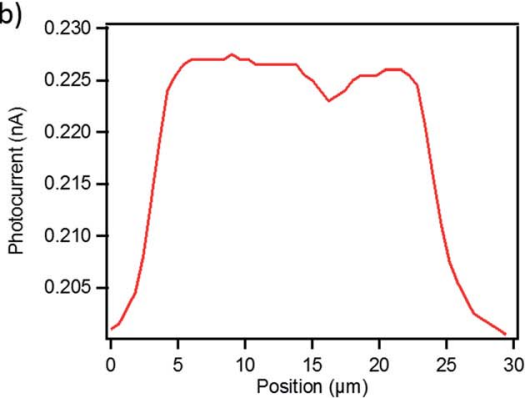

Fig. 10 (a) Zero drain bias photocurrent response for OFET with $24 \%$ GNR/P3HT and $L=20 \mu \mathrm{m}$ in the off-state $\left(V_{G}=+30\right.$ V). The dashed white lines indicate the edges of the source and drain electrical contacts; the black circle indicates the observed aggregate of GNRs. (b) Variation of the photocurrent as a function of the position of the black dashed line in (a).

to that at $605 \mathrm{~nm}$, we find that $R$ values are similar to those at $605 \mathrm{~nm}$ (Fig. S13a in the ESI†), whereas $P$ values are slightly higher (Fig. S13b in the ESI $\dagger$ ). Only a small increase in the photoresponse is observed by lowering the incident wavelength, which can be explained by (i) the greater energy of photons of $\lambda$ $=560 \mathrm{~nm}$ when compared to $\lambda=605 \mathrm{~nm}$ and (ii) the small difference in the absorbance peaks at $\lambda=560 \mathrm{~nm}$ and $605 \mathrm{~nm}$ (Fig. S12 in the ESI $\dagger$ ). For pristine P3HT devices, $R$ and $P$ for $L=$ $10 \mu \mathrm{m}$ are close to the ones reported in the literature. ${ }^{73,81}$ This suggests that the use of a monochromatic light source with a wavelength matching the peak of absorption of the semiconductor gives a higher photoresponse as compared to broadband illumination (see Fig. S13d in the ESI, $\uparrow$ where the same device is tested under white light and monochromatic light at 605 and $560 \mathrm{~nm}$ ). The field-effect mobility of the devices studied at both wavelengths remained relatively unaffected under illumination as compared to the one in the dark (see Fig. S13c in the ESI $\dagger$ ). Furthermore, after 5 min of switching off the light, both devices at 0 and $24 \%$ did not regain their initial characteristics due to a photoinduced memory effect (Fig. 8b and d green plots). In view of this finding, we extended our study to the examination of this slow relaxation at different blend percentages as a function of the gate voltage (with $V_{\mathrm{G}}=$ $0 \mathrm{~V}$ and without $V_{\mathrm{G}}$ ). Fig. 9a and b depict the relaxation mechanism of the photoinduced charges after illumination for $30 \mathrm{~s}$ for a $20 \mu \mathrm{m}$ device with 0 and $24 \%$ blend respectively, measured at $V_{\mathrm{D}}=-10 \mathrm{~V}$. In both cases, the photoinduced current decreases slowly once the light source is switched off at $t=60 \mathrm{~s}$ (see Fig. 9a and b) and in the same manner regardless of the application (blue plots) or not (red plots) of a gate bias. The same result was observed for the other blend percentages. This decay was reported for regioregular $\mathrm{P}_{3} \mathrm{HT}^{82,83}$ and fitted to a stretched-exponential behavior (Kohlrausch's law) (grey curves in Fig. 9a and b):

$$
\sigma(t)=\sigma_{0} \exp \left[-\left(\frac{t}{\tau}\right)^{\beta}\right] \quad 0<\beta<1
$$

where $\sigma$ is the conductivity, $\tau$ is the relaxation lifetime and the exponent $\beta$ indicates the degree of the disorder of the material. Fig. 9c portrays the average of the extracted values for $\beta$ for different blend percentages. It reveals that $\beta$ values are almost in the same range considering the error of the fitting and the error bars with or without $V_{\mathrm{G}}$. As for the variation of the relaxation lifetime $\tau$ with the concentration of GNRs, the results are shown in Fig. 9d. The average $\tau$ depends on the fitting parameters, blend \% and the gate bias. If no gate bias is applied, $\tau$ does not follow any trend with the blend $\%$ whereas at $V_{\mathrm{G}}=0 \mathrm{~V}$, when considering the error bars, there is no clear trend when increasing the blend \%. This can be attributed to the random distribution of the GNRs within the P3HT domains inside the channel, which will result in the variation of the trap densities, thus affecting $\tau$. It was shown that the relaxation mechanism depends on the bulk trap densities, the insulator-semiconductor interface, the diffusion rate of the photoinduced charge carriers, the gate voltage ${ }^{83}$ and mainly the presence of negatively charged electron accepting impurities ${ }^{82}$ present in P3HT. This might explain the difference observed with $\tau$ and makes it difficult to understand if the aggregates of GNRs help in increasing the recombination rate of holes.

\section{Photocurrent mapping}

To gain more information about the local origin of the generated photoresponse and the role of the GNRs under illumination, we studied these devices using scanning photocurrent microscopy. This technique has been employed widely for the local photocurrent study of devices based on carbon nanotubes and graphene, ${ }^{\mathbf{8 4 - 8 8}}$ and uses an optical microscope in combination with a multi-axis scanning stage for raster scanning the sample with respect to a laser beam focused on the device surface. We examined a device $(L=20 \mu \mathrm{m})$ with $24 \%$ GNRs blended with P3HT at $V_{\mathrm{G}}=+30 \mathrm{~V}$ (in the off-state) and at zero source-drain bias under a $\sim 1 \mu \mathrm{m}$ spot size laser $(\lambda=633 \mathrm{~nm}$, power density $<50 \mathrm{~kW} \mathrm{~cm}^{-2}$ ). The photocurrent image presented in Fig. 10 reveals uniform photocurrent response inside the channel indicating that the entire OFET channel is photoactive. However, a decrease in local photocurrent is observed in the areas where big aggregates of GNRs are present (see the black circle in Fig. 10a and the photocurrent profile in Fig. 10b). The difference could be explained by the fact that the photogenerated carriers in the aggregated region face higher impedance due to the potential barrier formed at the interface between the GNR aggregates $\left(E_{\mathrm{g}} \mathrm{GNR} 1=\sim 1.6 \mathrm{eV}\right)$ and P3HT $\left(E_{\mathrm{g}}\right.$ $\mathrm{P} 3 \mathrm{HT}=1.9-2.0 \mathrm{eV}$ ) due the difference in their band gap (Fig. S5 
in the ESI $\dagger$ ). Therefore this barrier results in a lower photocurrent collected on local illumination in this region of the channel. However, this small difference does not strongly affect the overall performance of our phototransistors as demonstrated through measurements by illuminating the entire device area.

\section{Conclusion}

In summary, we designed and synthesized $N=18$ armchair graphene nanoribbons (GNR 1) with a good dispersibility in organic solvents and an ionization energy matching that of P3HT. GNR 1 was blended with P3HT in a single step codeposition process. While monocomponent GNR films suffer from the presence of macroscopic crystals alternated by domain boundaries, thereby lacking in terms of extended percolation pathways for charge transport, we demonstrated that the addition of up to $24 \%$ of GNRs in a P3HT film enables improvement of percolation of charges in a $\mathrm{P} 3 \mathrm{HT}$ transistor. In particular, it is possible to obtain a three-fold increase in the field-effect mobility in a P3HT device by adding $24 \%$ GNRs, without altering the other relevant electronic characteristics, such as the $I_{\text {on }} / I_{\text {off }}$. This can be ascribed to the aggregates of GNRs acting as percolation paths for the charge carrier within the conduction channel by connecting the domains of the semiconductor film. Our P3HT - GNR blend, exhibiting an increased $\mu$ accompanied by an unaltered $I_{\mathrm{on}} / I_{\text {off }}$ is a step forward towards the enhancement of the performance of OFETs. The transistor's photoresponse in GNR-P3HT binary mixtures depends on the quantity of each component in the blend as well as on the channel length. This may allow the use of these devices in (opto) electronics, where light can function as an additional control parameter.

\section{Experimental section}

\section{Materials}

Poly(3-hexylthiophene) (P3HT) (Fig. 1b) (regioregularity $>99 \%$, $M_{\mathrm{w}} \sim 50000 \mathrm{~g} \mathrm{~mol}^{-1}$, Sepiolid P-200 from BASF) was used as purchased. Other materials were purchased from Fluka, Aldrich, Acros, ABCR, Merck, and other commercial suppliers and used as received unless otherwise specified.

1,3-Di(biphenyl-3-yl)propan-2-on (2). To a solution of 3(bromomethyl)-biphenyl (17.6 g, $71.2 \mathrm{mmol}), \mathrm{Fe}(\mathrm{CO})_{5}(7.3 \mathrm{~g}$, $37 \mathrm{mmol})$, and benzyltriethylammonium chloride $(0.54 \mathrm{~g}$, $1.9 \mathrm{mmol})$ in dichloromethane $(170 \mathrm{~mL})$ was added a solution of potassium hydroxide $(17.3 \mathrm{~g}, 307 \mathrm{mmol})$ in $\mathrm{H}_{2} \mathrm{O}(9.0 \mathrm{~mL})$, and the reaction mixture was refluxed overnight. After cooling down to room temperature the mixture was quenched with $\mathrm{HCl}$, and the organic phase was concentrated in vacuo. The crude product was first purified by silica gel column chromatography (eluent: $6 \%$ ethyl acetate/hexane) and then dissolved in dichloromethane and reprecipitated from methanol to give the title compound as colorless crystals (5.31 g, $14.7 \mathrm{mmol}, 41 \%$ yield): Mp: $104.3{ }^{\circ} \mathrm{C} ;{ }^{1} \mathrm{H}$ NMR $\left(500 \mathrm{MHz}\right.$, tetrahydrofuran- $\left.d_{8}, 303 \mathrm{~K}\right) \delta=$ $7.56(\mathrm{~d}, J=7.3 \mathrm{~Hz}, 4 \mathrm{H}), 7.47(\mathrm{~d}, J=7.8 \mathrm{~Hz}, 2 \mathrm{H}), 7.44(\mathrm{~s}, 2 \mathrm{H}), 7.37$ $(\mathrm{t}, J=7.7 \mathrm{~Hz}, 4 \mathrm{H}), 7.34(\mathrm{t}, J=7.7 \mathrm{~Hz}, 2 \mathrm{H}), 7.28(\mathrm{t}, J=7.4 \mathrm{~Hz}, 2 \mathrm{H})$, $7.16(\mathrm{~d}, J=7.6 \mathrm{~Hz}, 2 \mathrm{H}), 3.83(\mathrm{~s}, 4 \mathrm{H}) ;{ }^{13} \mathrm{C}$ NMR (125 MHz, 1,2tetrachloroethane- $\left.d_{2}, 303 \mathrm{~K}\right) \delta=205.65,141.34,140.40,134.21$, 129.06, 128.70, 128.41, 128.22, 127.39, 127.03, 125.81, 120.18, 49.14; MS (FD, $8 \mathrm{kV}): m / z(\%): 361.6$ (100) $\left[\mathrm{M}^{+}\right]$(calcd for $\left.\mathrm{C}_{27} \mathrm{H}_{22} \mathrm{O}: 362.2\right)$; Rf (30\% ethyl acetate/hexane) $=0.75$; anal. found: C, 89.5; $\mathrm{H}, 6.3$ (calcd for $\mathrm{C}_{27} \mathrm{H}_{22} \mathrm{O}$ : C, 89.5; $\mathrm{H}, 6.1 \%$ ).

2,5-Di([1,1'-biphenyl]-3-yl)-3,4-bis(4-chlorophenyl)cyclopenta2,4-dienone (4). To a degassed solution of 4,4'-didochlorobenzil $(3)^{89}$ (940 mg, $3.37 \mathrm{mmol}$ ) and 1,3-di(biphenyl-3-yl)propan-2-on (1) $(1.22 \mathrm{~g}, 3.37 \mathrm{mmol})$ in tert-butanol $(20 \mathrm{~mL})$ was added at $80{ }^{\circ} \mathrm{C}$ a methanol solution of tetrabutylammonium hydroxide (1.0 M, 1.7 mL, $1.7 \mathrm{mmol})$. After stirring at $80{ }^{\circ} \mathrm{C}$ for $20 \mathrm{~min}$ the reaction was quenched with water, and the reaction mixture was extracted three times with dichloromethane. The combined organic layers were washed with brine, dried over magnesium sulfate, and evaporated to give a purple crude product. Purification by silica gel column chromatography (eluent: $20 \%$ dichloromethane/hexane) gave the title compound as a purple solid (1.56 g, $2.58 \mathrm{mmol}$, 77\% yield): ${ }^{1} \mathrm{H}$ NMR (700 MHz, tetrahydrofuran- $\left.d_{8}\right) \delta=7.52(\mathrm{~s}, 2 \mathrm{H}, \mathrm{CH}), 7.50(\mathrm{~d}, J=7.8 \mathrm{~Hz}, 2 \mathrm{H}$, $\mathrm{CH}), 7.42$ (d, $J=7.2 \mathrm{~Hz}, 4 \mathrm{H}, \mathrm{CH}), 7.36(\mathrm{t}, J=7.7 \mathrm{~Hz}, 4 \mathrm{H}, \mathrm{CH})$, $7.32(\mathrm{~m}, 6 \mathrm{H}, \mathrm{CH}), 7.27(\mathrm{t}, J=7.3 \mathrm{~Hz}, 2 \mathrm{H}, \mathrm{CH}), 7.23(\mathrm{~d}, J=7.8 \mathrm{~Hz}$, $2 \mathrm{H}, \mathrm{CH}), 7.06$ (d, $J=8.5 \mathrm{~Hz}, 4 \mathrm{H}, \mathrm{CH}) ;{ }^{13} \mathrm{C}$ NMR $(175 \mathrm{MHz}$, tetrahydrofuran- $\left.d_{8}\right) \delta=199.86,154.28,141.91,141.82,135.61$, 133.14, 132.14, 132.10, 129.98, 129.90, 129.70, 129.69, 129.52, 128.27, 127.78, 127.20, 126.73; MS (MALDI-TOF, positive): $\mathrm{m} / \mathrm{z}$ : $604.6\left[\mathrm{M}^{+}\right]$(calcd for $\mathrm{C}_{41} \mathrm{H}_{26} \mathrm{Cl}_{2} \mathrm{O}$ : 604.1); $\mathrm{Rf}$ (10\% ethyl acetate) hexane $)=0.47$.

1,2-Bis(4-chlorophenyl)-3,6-bis(biphenyl-3-yl)-4,5-bis(4-dodecylphenyl)benzene (5). A degassed solution of $2,5-\mathrm{di}\left(\left[1,1^{\prime}-\right.\right.$ biphenyl]-3-yl)-3,4-bis(4-chlorophenyl)cyclopenta-2,4-dienone (4) (1.84 g, $3.03 \mathrm{mmol}$ ) and bis(4-dodecylphenyl)acetylene ${ }^{\mathbf{9 0}}$ (1.72 g, $3.34 \mathrm{mmol})$ in diphenyl ether $(12 \mathrm{~mL})$ and propylene carbonate $(5.0 \mathrm{~mL})$ was stirred at $230{ }^{\circ} \mathrm{C}$ for $12 \mathrm{~h}$ twice under microwave irradiation with a maximum power of $300 \mathrm{~W}$ and a maximum pressure of 7 bar. After cooling down to room temperature the reaction mixture was diluted with hexane and purified by silica gel column chromatography (eluent: 6\% ethyl acetate/hexane). The product was further purified by using recycling preparative SEC (Waters Ultrastyragel $103 \AA$ A, $19 \times 300 \mathrm{~mm}$, eluent: chloroform, $3.0 \mathrm{~mL} \mathrm{~min}^{-1}$ ) to give the title compound as colorless oil (1.85 g, $1.69 \mathrm{mmol}, 56 \%$ yield): ${ }^{1} \mathrm{H}$ NMR (700 MHz, tetrahydrofuran- $\left.d_{8}\right) \delta=7.27(\mathrm{dt}, J=7.7,4.0 \mathrm{~Hz}, 4 \mathrm{H}, \mathrm{CH}), 7.22-7.16(\mathrm{~m}$, $6 \mathrm{H}, \mathrm{CH}), 7.10(\mathrm{~d}, J=10.0 \mathrm{~Hz}, 4 \mathrm{H}, \mathrm{CH}), 6.94(\mathrm{t}, J=7.7 \mathrm{~Hz}, 4 \mathrm{H}$, $\mathrm{CH}), 6.91(\mathrm{~s}, 2 \mathrm{H}), 6.88(\mathrm{~d}, J=8.4 \mathrm{~Hz}, 2 \mathrm{H}, \mathrm{CH}), 6.87-6.81(\mathrm{~m}, 2 \mathrm{H}$, $\mathrm{CH}$ ), 6.81-6.77 (m, 2H, CH), 6.77-6.70 (br m, 6H, CH), 6.66 (d, $J=7.5 \mathrm{~Hz}, 2 \mathrm{H}, \mathrm{CH}), 2.41-2.28\left(\mathrm{~m}, 4 \mathrm{H}, \alpha-\mathrm{CH}_{2}\right), 1.44-1.34(\mathrm{~m}, 4 \mathrm{H}$, $\left.\beta-\mathrm{CH}_{2}\right), 1.34-1.03\left(\mathrm{~m}, 36 \mathrm{H},-\mathrm{CH}_{2}-\right), 0.89(t, J=6.9 \mathrm{~Hz}, 6 \mathrm{H}$, $\left.-\mathrm{CH}_{3}\right) ;{ }^{13} \mathrm{C}$ NMR $\left(175 \mathrm{MHz}\right.$, tetrahydrofuran- $\left.d_{8}\right) \delta=142.44$, 142.42, 142.27, 141.88, 141.74, 140.76, 140.74, 140.68, 140.59, 140.56, 140.14, 140.10, 139.09, 139.05, 134.18, 134.14, 134.06, 134.01, 132.56, 132.50, 132.47, 132.30, 132.26, 131.69, 131.41, 129.37, 128.20, 128.07, 127.90, 127.88, 127.84, 127.81, 127.80, 127.74, 125.14, 125.12, 36.36, 36.33, 33.06, 32.37, 32.34, 30.85, $30.81,30.77,30.75,30.61,30.60,30.51,30.00,29.98,25.94$, 25.82, 23.74, 14.62; MS (MALDI-TOF, positive): $\mathrm{m} / \mathrm{z}: 1091.0$ $\left[\mathrm{M}^{+}\right]$(calcd for $\mathrm{C}_{78} \mathrm{H}_{84} \mathrm{Cl}_{2}$ : 1090.6); $\mathrm{Rf}(6 \%$ ethyl acetate/hexane) 
$=0.65$; anal. found: $\mathrm{C}, 85.6 ; \mathrm{H}, 7.9$ (calcd for $\mathrm{C}_{78} \mathrm{H}_{84} \mathrm{Cl}_{2}: \mathrm{C}$, 85.8; H, 7.8\%).

Polyphenylene precursor 6. The catalytic system consisting of $\mathrm{Ni}(\mathrm{COD})_{2}$ (589 mg, $\left.2.14 \mathrm{mmol}\right), 1,5$-cyclooctadiene (232 mg, $2.14 \mathrm{mmol}$ ), and 2,2'-bipyridine (334 $\mathrm{mg}, 2.14 \mathrm{mmol}$ ) in a mixture of DMF $(2.0 \mathrm{~mL})$ and toluene $(2.0 \mathrm{~mL})$ was prepared in a glove box under a nitrogen atmosphere and wrapped in aluminum foil for the exclusion of light. The catalyst system was activated by stirring at $60{ }^{\circ} \mathrm{C}$ for $20 \mathrm{~min}$, and then a degassed solution of 1,2-bis(4-chlorophenyl)-3,6-bis(biphenyl-3-yl)-4,5bis(4-dodecylphenyl)benzene (5) (975 mg, $0.893 \mathrm{mmol}$ ) in dry toluene $(2.0 \mathrm{~mL})$ was added. The reaction mixture was stirred at $80{ }^{\circ} \mathrm{C}$ for 10 days. For endcapping of the resulting polymer an excess amount of chlorobenzene was added and heated for additional 20 minutes at $80{ }^{\circ} \mathrm{C}$. After cooling down to room temperature the reaction mixture was added dropwise to a mixture of hydrochloric acid and methanol $(1: 10)$, and stirred overnight. The precipitates were collected by centrifugation and then repeatedly reprecipitated from THF solution in methanol to give the title compound as a white solid (780 mg, 86\% yield). A part of the polymer was fractionated by preparative SEC (BioBeads S-X1 support, Bio-Rad Laboratories), and then further by recycling preparative SEC to obtain a polymer fraction with $M_{\mathrm{w}}=1.0 \times 10^{4} \mathrm{~g} \mathrm{~mol}^{-1}, M_{\mathrm{n}}=7.2 \times 10^{3} \mathrm{~g} \mathrm{~mol}^{-1}$, and PDI $=1.4$ as well as a fraction with $M_{\mathrm{w}}=1.2 \times 10^{4} \mathrm{~g} \mathrm{~mol}^{-1}, M_{\mathrm{n}}=9.3 \times$ $10^{3} \mathrm{~g} \mathrm{~mol}^{-1}$, and PDI $=1.3$ based on SEC analysis (UV detector, polystyrene standard): FTIR (powder): 4052, 3084, 3054, 3029, 2924, 2852, 1598, 1573, 1513, 1498, 1479, 1465, 1451, 1407, 1356, 1259, 1184, 1141, 1118, 1092, 1074, 1053, 1021, 1006, 965, 944, 897, 841, 807, 717, $698 \mathrm{~cm}^{-1}$.

GNR 1. A solution of polyphenylene precursor 6 (76.6 $\mathrm{mg})$ in unstabilized dichloromethane $(80 \mathrm{~mL})$ was degassed by argon bubbling for $15 \mathrm{~min}$. To the solution was added a suspension of iron(III) chloride $(1.10 \mathrm{~g}, 6.76 \mathrm{mmol})$ in nitromethane $(3.5 \mathrm{~mL})$. After stirring at room temperature for 3 days with a continuous argon flow through the reaction mixture, the reaction was quenched by the addition of methanol to form black precipitates, which were collected by centrifugation. The crude product was intensively washed with THF and methanol using a Soxhlet extractor for 2 days to give the title compound as a black powder (72.9 mg, 98\% yield): FTIR (powder): 2920, 2850, 1595, 1455, 1372, 864, 823, 802, 759, 740, $720 \mathrm{~cm}^{-1}$; Raman (powder, 488 $\mathrm{nm}): 3200,2922,2725,1578,1325 \mathrm{~cm}^{-1}$.

\section{Solid-state NMR analysis}

All solid-state ${ }^{1} \mathrm{H}$ NMR experiments were carried out on a Bruker AVANCE III 700 operating at a Larmor frequency of $700.21 \mathrm{MHz}$ $(16.45 \mathrm{~T})$. The experiments employed a $1.3 \mathrm{~mm} \mathrm{H}-\mathrm{X}$ doubleresonance magic-angle spinning (MAS) probe from Bruker, using a spinning frequency of $59524 \mathrm{~Hz}$ and a $\pi / 2$-pulse length of $1.5 \mu \mathrm{s}$, corresponding to an rf-field strength of $167 \mathrm{kHz}$. The $2 \mathrm{D}{ }^{1} \mathrm{H}-{ }^{1} \mathrm{H}$ double quantum-single quantum (DQ-SQ) correlation experiments were carried out using the compensated Back-toBack $(\mathrm{BaBa})$ dipolar recoupling sequence for both excitation and reconversion of DQ coherences followed by a z-filter, set to one rotor period, prior to a final $\pi / 2$ pulse for creating transverse observable magnetization. ${ }^{62,63}$ A phase cycling scheme of 16 steps, a recycle delay of $2 \mathrm{~s}$, and 16 scans per rotorsynchronized $t_{1}$ increment were used. Prior to all experiments the magic angle was checked with $\mathrm{KBr} .{ }^{91}{ }^{1} \mathrm{H}$ chemical shifts are reported relative to TMS using solid adamantane as an external ref. 92 The $2 \mathrm{D}{ }^{1} \mathrm{H}-{ }^{1} \mathrm{H}$ DQ-SQ spectra shown in Fig. 4 were processed identically and plotted on the same intensity scale with 22 contour lines from $2.0 \%$ to $90 \%$ of the maximum intensity.

\section{Device fabrication}

Bottom-gate bottom-contact transistors exposing $230 \mathrm{~nm}$ thermally grown oxide on $\mathrm{n}++$-doped silicon (Fraunhofer Institute, capacitance $1.5 \times 10^{-8} \mathrm{~F} \mathrm{~cm}^{-2}$ ) were used. Each substrate exposes prepatterned interdigitated Au source-drain electrodes with different channel lengths $(L=2.5,5,10$, and $20 \mu \mathrm{m})$ and constant channel width $(W=10 \mathrm{~mm})$. These substrates were cleaned in an ultrasonic bath of acetone and isopropanol prior to device fabrication. For the devices with higher channel lengths $(L=60,80,100$, and $120 \mu \mathrm{m})$ and same width $(W=10$ $\mathrm{mm}$ ), interdigitated Au source-drain electrodes (40 nm) were evaporated (chamber pressure $=10^{-6} \mathrm{mbar}$, evaporation rate $=$ $0.03 \mathrm{~nm} \mathrm{~s}^{-1}$ ) on bare $\mathrm{SiO}_{x}$ substrates (having the same specifications as above mentioned) using a shadow mask. GNRs were dispersed in ODCB and sonicated for at least 90 min then immediately transferred to the glove box $\left(\mathrm{N}_{2}\right.$ atmosphere) where they were mixed with a constant amount of P3HT $1.5 \mathrm{mg} \mathrm{mL}^{-1}$ in ODCB) at different percentages $(2,5,11$ and $24 \mathrm{wt} \%$ with respect to $\mathrm{P} 3 \mathrm{HT}$ ). Thin films were prepared inside the glovebox $\left(\mathrm{N}_{2}\right.$ atmosphere) by spin-coating the blend at $1200 \mathrm{rpm}$ for $60 \mathrm{~s}$ onto untreated substrates. This was followed by an annealing step at $200{ }^{\circ} \mathrm{C}$ for $15 \mathrm{~min}$. For comparison, we prepared pristine $\mathrm{P} 3 \mathrm{HT}$ devices that were processed in the same fashion as the blended ones.

\section{Electrical characterization of the devices}

Electrical characterization of the devices was performed at room temperature in a $\mathrm{N}_{2}$ atmosphere inside a glovebox, using a Cascade Microtech M150 probe station and a Keithley 2636A sourcemeter as a semiconductor parameter analyzer controlled by associated software.

To study the photoresponse, at least 6 devices for each channel length and each blend percentage were characterized under illumination from the top using a Polychrome V (Till Photonics) tunable light source providing a monochromatic beam with $605 \mathrm{~nm}$ and $560 \mathrm{~nm}$ wavelengths and irradiance levels of 8.17 and $9.44 \mathrm{~mW} \mathrm{~cm}^{-2}$ respectively. The light intensity was measured using an analog optical power meter, PM100A (ThorLabs). These wavelengths were chosen in view of the absorbance spectra and correspond to two absorption peaks of the P3HT films as shown in Fig. S12 in the ESI. $\dagger$ The measurement sequence used is as follows: two consecutive measurements for transfer and output characteristics in the dark. The light is then switched on and transfer and output characteristics under illumination are measured. For some devices only, this was followed by measurements in the dark 
after $5 \mathrm{~min}$ of switching off the light to see if there is a photoinduced memory effect.

To study the relaxation of the photo-induced charges, 5 to 6 devices of $L=20 \mu \mathrm{m}$ for each blend percentage were characterized under illumination at $\lambda=560 \mathrm{~nm}\left(9.44 \mathrm{~mW} \mathrm{~cm}^{-2}\right)$. Two measurement sequences were used: in the first case, continuous gate bias $V_{\mathrm{G}}=0 \mathrm{~V}$ and drain bias $V_{\mathrm{D}}=-10 \mathrm{~V}$ were applied in the dark for $30 \mathrm{~s}$ then the light is switched on for $30 \mathrm{~s}$ then switched off for $30 \mathrm{~s}$ whereas in the second case the same was followed except that the gate was floating (no gate bias applied).

\section{Photocurrent mapping}

Photocurrent mapping was done using a laser light $(\lambda=633 \mathrm{~nm}$, power density $<50 \mathrm{~kW} \mathrm{~cm}^{-2}$ ) generated from a He-Ne laser which was coupled to the sample by using a DM LM microscope (Leica) and a $\times 100$ ultra-long working distance objective, resulting in a $\sim 1 \mu \mathrm{m}$ spot size. A Physik Instrument piezoelectric stage was used to translate the device with respect to the laser spot in the $x /$ $y$ directions with $500 \mathrm{~nm}$ steps, resulting in confocal, positiondependent recording of the generated photocurrent. Measurements were done at room temperature with a low pressure $\mathrm{N}_{2}$ flow over the sample. Photocurrent signals were recorded by modulating the laser beam $(1 \mathrm{kHz})$ using a mechanical chopper and the short circuit photocurrent was detected by using a lock-in amplifier. A 2400 Sourcemeter (Keithley) was employed to control the gate voltage. For this experiment, in order to overcome the air sensitivity issue of P3HT, an encapsulating film of poly(methyl methacrylate) (PMMA) (70 $\mathrm{mg} \mathrm{mL}^{-1}$ in methyl ethyl ketone) was spin coated onto the devices at $1000 \mathrm{rpm}$ for $30 \mathrm{~s}, 200 \mu \mathrm{L}$ (see ESI $\dagger$ for more details).

\section{Instrumentation}

AFM images were recorded using a Nanoscope (Veeco Multimode V). Measurements of the thickness of the active layer were performed using an Alpha step IQ profiler. Scanning electron microscopy (SEM) images for pure GNRs were taken using a Strata 400 Dual Beam. Samples were prepared by drop casting (0.25 $\mathrm{mg} \mathrm{mL}^{-1}$ in ODCB) followed by annealing at $200{ }^{\circ} \mathrm{C}$ for 15 min then sputtering with $\mathrm{Au}(30 \mathrm{~s}, I=60 \mathrm{~mA})$. Ambient photoelectron spectroscopy measurements were performed on drop cast films (annealed at $200{ }^{\circ} \mathrm{C}$ for $15 \mathrm{~min}$ inside the glovebox) using photoelectron spectroscopy operating under atmospheric conditions (RIKEN AC-2). The same instrument was used for determining the work function of $\mathrm{Au}$ thin films prepared by vacuum evaporation (at $1.7 \times 10^{-6} \mathrm{mbar}$ ) onto mica substrates $\left(450{ }^{\circ} \mathrm{C}\right)$. X-ray diffraction (GIXD) measurements were performed using the grazing-incidence geometry. The experimental setup included a rotating copper anode X-ray source (Rigaku Micromax, operating at $42 \mathrm{kV}$ and $20 \mathrm{~mA}$ ), Osmic confocal MaxFlux optics and a three $x / y$-adjustable pinhole collimation system (JJ X-ray). Samples deposited on top of silicone substrates were irradiated with a wavelength of $\lambda=$ $1.5418 \AA$ at the incident angle $\left(\alpha_{\mathrm{i}}\right)$ of $0.11^{\circ}$. The GIXD patterns were recorded for $5 \mathrm{~h}$ on a MAR345 image plate detector. Camera length $(315 \mathrm{~mm})$ and the range of detectable $d$-spacings $\left(\sim 35 \AA<d_{h k l}<3 \AA\right)$ were calibrated using the silver behenate standard. The data were processed using the Datasqueeze 2.2.9 program.

\section{Acknowledgements}

We acknowledge funding by the EC - Marie-Curie ITNs GENIUS (PITN-GA-2010-264694) and SUPERIOR (PITN-GA-2009-238177), the Graphene Flagship (GA-604391), the International Center for Frontier Research in Chemistry (icFRC), the Marie Curie project (PIEF-GA-2009-253521), the statutory activity subsidy for the Faculty of Chemistry of Wroclaw University of Technology, ERC Grants NANOPOTS and Hetero2D, EPSRC Grants EP/ K01711X/1, EP/K017144/1, EP/L016087/1, EU grant CARERAMM, MEM4WIN, a Royal Society Wolfson Research Merit Award. MRH acknowledges financial support from the Villum Foundation under the Young Investigator Programme (VKR023122).

\section{References}

1 H. Yan, Z. Chen, Y. Zheng, C. Newman, J. R. Quinn, F. Dotz, M. Kastler and A. Facchetti, Nature, 2009, 457, 679.

2 B. C. Schroeder, C. B. Nielsen, Y. J. Kim, J. Smith, Z. Huang, J. Durrant, S. E. Watkins, K. Song, T. D. Anthopoulos and I. McCulloch, Chem. Mater., 2011, 23, 4025.

3 I. McCulloch, R. S. Ashraf, L. Biniek, H. Bronstein, C. Combe, J. E. Donaghey, D. I. James, C. B. Nielsen, B. C. Schroeder and W. Zhang, Acc. Chem. Res., 2012, 45, 714.

4 J. E. Anthony, Chem. Rev., 2006, 106, 5028.

5 J. E. Anthony, A. Facchetti, M. Heeney, S. R. Marder and X. W. Zhan, Adv. Mater., 2010, 22, 3876.

6 A. C. Arias, J. D. MacKenzie, I. McCulloch, J. Rivnay and A. Salleo, Chem. Rev., 2010, 110, 3.

7 A. Facchetti, Mater. Today, 2007, 10, 28.

8 I. McCulloch, M. Heeney, C. Bailey, K. Genevicius, M. I. MacDonald, M. Shkunov, D. Sparrowe, S. Tierney, R. Wagner, W. M. Zhang, M. L. Chabinyc, R. J. Kline, M. D. Mcgehee and M. F. Toney, Nat. Mater., 2006, 5, 328.

9 X. W. Zhan, A. Facchetti, S. Barlow, T. J. Marks, M. A. Ratner, M. R. Wasielewski and S. R. Marder, Adv. Mater., 2011, 23, 268. 10 M. Zhang, H. N. Tsao, W. Pisula, C. D. Yang, A. K. Mishra and K. Müllen, J. Am. Chem. Soc., 2007, 129, 3472.

11 J. Smith, R. Hamilton, Y. Qi, A. Kahn, D. D. C. Bradley, M. Heeney, I. McCulloch and T. D. Anthopoulos, Adv. Funct. Mater., 2010, 20, 2330.

12 E. Orgiu, A. M. Masillamani, J.-O. Vogel, E. Treossi, A. Kiersnowski, M. Kastler, W. Pisula, F. Dotz, V. Palermo and P. Samorì, Chem. Commun., 2012, 48, 1562.

13 R. Dabirian, V. Palermo, A. Liscio, E. Schwartz, M. B. J. Otten, C. E. Finlayson, E. Treossi, R. H. Friend, G. Calestani, K. Müllen, R. J. M. Nolte, A. E. Rowan and P. Samori, J. Am. Chem. Soc., 2009, 131, 7055.

14 J. Smith, W. Zhang, R. Sougrat, K. Zhao, R. Li, D. Cha, A. Amassian, M. Heeney, I. McCulloch and T. D. Anthopoulos, Adv. Mater., 2012, 24, 2441.

15 R. Hamilton, J. Smith, S. Ogier, M. Heeney, J. E. Anthony, I. McCulloch, J. Veres, D. D. C. Bradley and T. D. Anthopoulos, Adv. Mater., 2009, 21, 1166. 
16 E. Lim, B. J. Jung, M. Chikamatsu, R. Azumi, K. Yase, L. M. Do and H. K. Shim, Org. Electron., 2008, 9, 952.

17 D. M. Russell, C. J. Newsome, S. P. Li, T. Kugler, M. Ishida and T. Shimoda, Appl. Phys. Lett., 2005, 87, 222109.

18 J. Smith, R. Hamilton, M. Heeney, D. M. de Leeuw, E. Cantatore, J. E. Anthony, I. McCulloch, D. D. C. Bradley and T. D. Anthopoulos, Appl. Phys. Lett., 2008, 93, 253301.

19 J. Smith, R. Hamilton, Y. B. Qi, A. Kahn, D. D. C. Bradley, M. Heeney, I. McCulloch and T. D. Anthopoulos, Adv. Funct. Mater., 2010, 20, 2330.

20 E. Orgiu, N. Crivillers, M. Herder, L. Grubert, M. Pätzel, J. Frisch, E. Pavlica, D. T. Duong, G. Bratina, A. Salleo, N. Koch, S. Hecht and P. Samori, Nat. Chem., 2012, 4, 675.

21 K.-J. Baeg, D. Khim, D.-Y. Kim, J. B. Koo, I.-K. You, W. S. Choi and Y.-Y. Noh, Thin Solid Films, 2010, 518, 4024.

22 Z. Bao, A. Dodabalapur and A. J. Lovinger, Appl. Phys. Lett., 1996, 69, 4108.

23 Y. Fu, C. Lin and F.-Y. Tsai, Org. Electron., 2009, 10, 883.

24 M. Surin, P. Leclere, R. Lazzaroni, J. D. Yuen, G. Wang, D. Moses, A. J. Heeger, S. Cho and K. Lee, J. Appl. Phys., 2006, 100, 033712.

25 H. Sirringhaus, P. J. Brown, R. H. Friend, M. M. Nielsen, K. Bechgaard, B. M. W. Langeveld-Voss, A. J. H. Spiering, R. A. J. Janssen, E. W. Meijer, P. Herwig and D. M. de Leeuw, Nature, 1999, 401, 685.

26 J. Huang, D. R. Hines, B. J. Jung, M. S. Bronsgeest, A. Tunnell, V. Ballarotto, H. E. Katz, M. S. Fuhrer, E. D. Williams and J. Cumings, Org. Electron., 2011, 12, 1471.

27 A. Liscio, G. P. Veronese, E. Treossi, F. Suriano, F. Rossella, V. Bellani, R. Rizzoli, P. Samorì and V. Palermo, J. Mater. Chem., 2011, 21, 2924.

28 X. Z. Bo, C. Y. Lee, M. S. Strano, M. Goldfinger, C. Nuckolls and G. B. Blanchet, Appl. Phys. Lett., 2005, 86, 182102.

29 Y. D. Park, J. A. Lim, Y. Jang, M. Hwang, H. S. Lee, D. H. Lee, H.-J. Lee, J.-B. Baek and K. Cho, Org. Electron., 2008, 9, 317.

30 K. S. Novoselov, A. K. Geim, S. V. Morozov, D. Jiang, Y. Zhang, S. V. Dubonos, I. V. Grigorieva and A. A. Firsov, Science, 2004, 306, 666.

31 C. Mattevi, F. Colléaux, H. Kim, Y. H. Lin, K. T. Park, M. Chhowalla and T. D. Anthopoulos, Nanotechnology, 2012, 23, 344017.

32 C.-A. Palma and P. Samorì, Nat. Chem., 2011, 3, 431.

33 L. Chen, Y. Hernandez, X. Feng and K. Müllen, Angew. Chem., Int. Ed., 2012, 51, 7640.

34 M. Y. Han, B. Özyilmaz, Y. Zhang and P. Kim, Phys. Rev. Lett., 2007, 98, 206805.

35 B. Ozyilmaz, P. Jarillo-Herrero, D. Efetov and P. Kim, Appl. Phys. Lett., 2007, 91, 192107.

36 D. Yoon, H. Moon, H. Cheong, J. S. Choi, J. A. Choi and B. H. Park, J. Korean Phys. Soc., 2009, 55, 1299.

37 L. Jiao, L. Zhang, X. Wang, G. Diankov and H. Dai, Nature, 2009, 458, 877.

38 D. V. Kosynkin, A. L. Higginbotham, A. Sinitskii, J. R. Lomeda, A. Dimiev, B. K. Price and J. M. Tour, Nature, 2009, 458, 872 .
39 T. Shimizu, J. Haruyama., D. C. Marcano, D. V. Kosinkin, J. M. Tour, K. Hirose. and K. Suenaga., Nat. Nanotechnol., 2011, 6, 45.

40 X. Li, X. Wang, L. Zhang, S. Lee and H. Dai, Science, 2008, 319, 1229.

41 L. Dössel, L. Gherghel, X. Feng and K. Müllen, Angew. Chem., Int. Ed., 2011, 50, 2540.

42 M. G. Schwab, A. Narita, Y. Hernandez, T. Balandina, K. S. Mali, S. De Feyter, X. Feng and K. Müllen, J. Am. Chem. Soc., 2012, 134, 18169.

$43 \mathrm{~J} . \mathrm{Wu}$, L. Gherghel, M. D. Watson, J. Li, Z. Wang, C. D. Simpson, U. Kolb and K. Müllen, Macromolecules, 2003, 36, 7082.

44 X. Yang, X. Dou, A. Rouhanipour, L. Zhi, H. J. Räder and K. Müllen, J. Am. Chem. Soc., 2008, 130, 4216.

45 J. Cai, P. Ruffieux, R. Jaafar, M. Bieri, T. Braun, S. Blankenburg, M. Muoth, A. P. Seitsonen, M. Saleh, X. Feng, K. Müllen and R. Fasel, Nature, 2010, 466, 470.

46 P. Rempala, J. Kroulík and B. T. King, J. Am. Chem. Soc., 2004, 126, 15002.

47 R. Scholl and C. Seer, Justus Liebigs Ann. Chem., 1912, 394, 111.

48 S. Osella, A. Narita, M. G. Schwab, Y. Hernandez, X. Feng, K. Müllen and D. Beljonne, ACS Nano, 2012, 6, 5539.

49 T. Yamamoto, Prog. Polym. Sci., 1992, 17, 1153.

50 T. Yamamoto, Bull. Chem. Soc. Jpn., 1999, 72, 621.

51 J. Sakamoto, M. Rehahn, G. Wegner and A. D. Schlüter, Macromol. Rapid Commun., 2009, 30, 653.

52 A. Narita, X. Feng, Y. Hernandez, S. A. Jensen, M. Bonn, H. Yang, I. A. Verzhbitskiy, C. Casiraghi, M. R. Hansen, A. H. R. Koch, G. Fytas, O. Ivasenko, B. Li, K. S. Mali, T. Balandina, S. Mahesh, S. De Feyter and K. Müllen, Nat. Chem., 2014, 6, 126.

53 K. Martin, J. Spickermann, H. J. Räder and K. Müllen, Rapid Commun. Mass Spectrom., 1996, 10, 1471.

54 A. Centrone, L. Brambilla, T. Renouard, L. Gherghel, C. Mathis, K. Müllen and G. Zerbi, Carbon, 2005, 43, 1593.

55 Z. B. Shifrina, M. S. Averina, A. L. Rusanov, M. Wagner and K. Müllen, Macromolecules, 2000, 33, 3525.

56 J. Shu, D. Dudenko, M. Esmaeili, J. H. Park, S. R. Puniredd, J. Y. Chang, D. W. Breiby, W. Pisula and M. R. Hansen, J. Am. Chem. Soc., 2013, 135, 11075.

57 S. P. Brown, Solid State Nucl. Magn. Reson., 2012, 41, 1.

58 N. Tasios, C. Grigoriadis, M. R. Hansen, H. Wonneberger, C. Li, H. W. Spiess, K. Müllen and G. Floudas, J. Am. Chem. Soc., 2010, 132, 7478.

59 A. Bohle, G. Brunklaus, M. R. Hansen, T. W. Schleuss, A. F. M. Kilbinger, J. Seltmann and H. W. Spiess, Macromolecules, 2010, 43, 4978.

60 M. R. Hansen, X. Feng, V. Macho, K. Müllen, H. W. Spiess and G. Floudas, Phys. Rev. Lett., 2011, 107, 257801.

61 M. M. Elmahdy, X. Dou, M. Mondeshki, G. Floudas, H.-J. Butt, H. W. Spiess and K. Müllen, J. Am. Chem. Soc., 2008, 130, 5311.

62 K. Saalwächter, F. Lange, K. Matyjaszewski, C.-F. Huang and R. Graf, J. Magn. Reson., 2011, 212, 204. 
63 M. Feike, D. E. Demco, R. Graf, J. Gottwald, S. Hafner and H. W. Spiess, J. Magn. Reson., Ser. A, 1996, 122, 214. 64 D. Sebastiani and K. N. Kudin, ACS Nano, 2008, 2, 661.

65 Z. Chen, C. S. Wannere, C. Corminboeuf, R. Puchta and P. v. R. Schleyer, Chem. Rev., 2005, 105, 3842.

66 F. Torrisi, T. Hasan, W. Wu, Z. Sun, A. Lombardo, T. S. Kulmala, G.-W. Hsieh, S. Jung, F. Bonaccorso, P. J. Paul, D. Chu and A. C. Ferrari, ACS Nano, 2012, 6, 2992. 67 D. M. Russell, C. J. Newsome, S. P. Li, T. Kugler, M. Ishida and T. Shimoda, Appl. Phys. Lett., 2005, 87, 222109.

68 D. Dudenko, A. Kiersnowski, J. Shu, W. Pisula, D. Sebastiani, H. W. Spiess and M. R. Hansen, Angew. Chem., Int. Ed., 2012, 51, 11068.

69 N. Kayunkid, S. Uttiya and M. Brinkmann, Macromolecules, 2010, 43, 4961.

70 K. Rahimi, I. Botiz, N. Stingelin, N. Kayunkid, M. Sommer, F. P. V. Koch, H. Nguyen, O. Coulembier, P. Dubois, M. Brinkmann and G. Reiter, Angew. Chem., Int. Ed., 2012, 51, 11131.

71 X. Wang, Y. Ouyang, X. Li, H. Wang, J. Guo and H. Dai, Phys. Rev. Lett., 2008, 100, 206803.

72 K. T. Kim, J. W. Jung and W. H. Jo, Carbon, 2013, 63, 202.

73 T. Pal, M. Arif and S. Khondaker, Nanotechnology, 2010, 21, 325201.

74 M. EL Gemayel, M. Treier, C. Musumeci, C. Li, K. Müllen and P. Samorì, J. Am. Chem. Soc., 2012, 134, 2429.

75 M. J. Deen and M. H. Kazemeini, Proc. IEEE, 2005, 93, 1312. 76 M. C. Hamilton and J. Kanicki, IEEE J. Sel. Top. Quantum Electron., 2004, 10, 840.

77 M. C. Hamilton, S. Martin and J. Kanicki, IEEE Trans. Electron Devices, 2004, 51, 877.
78 N. Marjanović, T. B. Singh, G. Dennler, S. Günes, H. Neugebauer, N. S. Sariciftci, R. Schwödiauer and S. Bauer, Org. Electron., 2006, 7, 188.

79 T. P. I. Saragi, M. Fetten and J. Salbeck, Appl. Phys. Lett., 2007, 90, 253506.

80 K. Wasapinyokul, W. I. Milne and D. P. Chu, J. Appl. Phys., 2011, 109, 084510.

$81 R=150 \mathrm{~A} \mathrm{~W}^{-1}$ (at $V_{\mathrm{G}}=-80 \mathrm{~V}$ ) and $P=103$ for $(L=3 \mu \mathrm{m})$ prepared by drop casting and studied under white light (51 $\mathrm{mW} \mathrm{cm}^{-2}$ ).

82 G. Dicker, M. P. de Haas, D. M. de Leeuw and L. D. A. Siebbeles, Chem. Phys. Lett., 2005, 402, 370.

83 S. Dutta and K. S. Narayan, Phys. Rev. B: Condens. Matter Mater. Phys., 2003, 68, 125208.

84 M. Burghard and A. Mews, ACS Nano, 2012, 6, 5752.

85 M. Engel, M. Steiner, R. S. Sundaram, R. Krupke, A. A. Green, M. C. Hersam and P. Avouris, ACS Nano, 2012, 6, 7303.

86 R. S. Sundaram, M. Steiner, H. Y. Chiu, M. Engel, A. A. Bol, R. Krupke, M. Burghard, K. Kern and P. Avouris, Nano Lett., 2011, 11, 3833.

87 E. J. H. Lee, K. Balasubramanian, R. T. Weitz, M. Burghard and K. Kern, Nat. Nanotechnol., 2008, 3, 486.

88 E. J. H. Lee, K. Balasubramanian, J. Dorfmuller, R. Vogelgesang, N. Fu, A. Mews, M. Burghard and K. Kern, Small, 2007, 3, 2038.

89 D. C. Neckers and G. Hauck, J. Org. Chem., 1983, 48, 4691.

90 T. Izawa, E. Miyazaki and K. Takimiya, Chem. Mater., 2009, 21, 903.

91 J. S. Frye and G. E. Maciel, J. Magn. Reson., 1982, 48, 125.

92 S. Hayashi and K. Hayamizu, Bull. Chem. Soc. Jpn., 1991, 64, 685. 